\title{
Article \\ Mitochondrial Dysfunction and Alterations in Mitochondrial Permeability Transition Pore (mPTP) Contribute to Apoptosis Resistance in Idiopathic Pulmonary Fibrosis Fibroblasts
}

\author{
Erika Rubí Luis-García ${ }^{1}$, Carina Becerril ${ }^{1}$, Alfonso Salgado-Aguayo ${ }^{2}$, Omar Emiliano Aparicio-Trejo ${ }^{3}$,

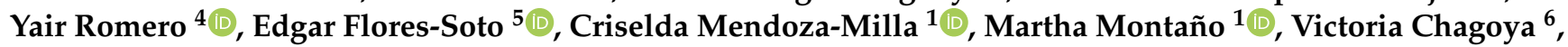 \\ José Pedraza-Chaverri ${ }^{3}{ }^{(1)}$, Mohammed El Hafidi ${ }^{7}$, Marisol Orozco-Ibarra ${ }^{8}\left[\right.$, , Annie Pardo ${ }^{4}$ (D) \\ and Moisés Selman $1, * \mathbb{C}$
}

check for

updates

Citation: Luis-García, E.R.; Becerril,

C.; Salgado-Aguayo, A.;

Aparicio-Trejo, O.E.; Romero, Y.;

Flores-Soto, E.; Mendoza-Milla, C.;

Montaño, M.; Chagoya, V.;

Pedraza-Chaverri, J.; et al.

Mitochondrial Dysfunction and

Alterations in Mitochondrial

Permeability Transition Pore (mPTP)

Contribute to Apoptosis Resistance in Idiopathic Pulmonary Fibrosis

Fibroblasts. Int. J. Mol. Sci. 2021, 22,

7870. https://doi.org/10.3390/

ijms22157870

Academic Editor: Paola Perego

Received: 3 June 2021

Accepted: 17 July 2021

Published: 23 July 2021

Publisher's Note: MDPI stays neutral with regard to jurisdictional claims in published maps and institutional affiliations.

Copyright: (c) 2021 by the authors. Licensee MDPI, Basel, Switzerland. This article is an open access article distributed under the terms and conditions of the Creative Commons Attribution (CC BY) license (https:// creativecommons.org/licenses/by/ $4.0 /)$.
1 Laboratorio de Biología Celular, Departamento de Fibrosis Pulmonar, Instituto Nacional de Enfermedades Respiratorias "Ismael Cosío Villegas", Ciudad de México 14080, Mexico; erikarubi.84@gmail.com (E.R.L.-G.); lcbb6@hotmail.com (C.B.); criselda.mendoza@gmail.com (C.M.-M.); mamora572002@yahoo.com.mx (M.M.)

2 Laboratorio de Investigación en Enfermedades Reumáticas, Instituto Nacional de Enfermedades Respiratorias "Ismael Cosío Villegas", Ciudad de México 14080, Mexico; alfonso.salgado@iner.gob.mx

3 Departamento de Biología, Facultad de Química, Universidad Nacional Autónoma de México, Ciudad de México 04510, Mexico; emilianoaparicio91@gmail.com (O.E.A.-T.); pedraza@unam.mx (J.P.-C.)

4 Facultad de Ciencias, Universidad Nacional Autónoma de México, Ciudad de México 04510, Mexico; yair12@hotmail.com (Y.R.); apardos@unam.mx (A.P.)

5 Departamento de Farmacología, Facultad de Medicina, Universidad Nacional Autónoma de México, Ciudad de México 04510, Mexico; edgarfloressoto@yahoo.com.mx

6 Departamento de Biología Celular y Desarrollo, Instituto de Fisiología Celular, Universidad Nacional Autónoma de México, Ciudad de México 04510, Mexico; vchagoya@ifc.unam.mx

7 Departamento de Biomedicina Cardiovascular, Instituto Nacional de Cardiología, Ignacio Chávez, Juan Badiano 1, Belisario Domínguez Secc 16, Tlalpan, Ciudad de México 14080, Mexico; medelhafidi@yahoo.com

8 Laboratorio de Neurobiología Molecular y Celular, Instituto Nacional de Neurología y Neurocirugía, Manuel Velasco Suárez, Av. Insurgentes Sur 3877, Colonia La Fama, Alcaldía Tlalpan, Ciudad de México 14269, Mexico; marisol.orozco.ibarra@gmail.com

* Correspondence: mselmanl@yahoo.com.mx; Tel.: +55-5-487-1700

\begin{abstract}
Idiopathic pulmonary fibrosis (IPF) is a devastating disease characterized by increased activation of fibroblasts / myofibroblasts. Previous reports have shown that IPF fibroblasts are resistant to apoptosis, but the mechanisms remain unclear. Since inhibition of the mitochondrial permeability transition pore (mPTP) has been implicated in the resistance to apoptosis, in this study, we analyzed the role of mitochondrial function and the MPTP on the apoptosis resistance of IPF fibroblasts under basal conditions and after mitomycin C-induced apoptosis. We measured the release of cytochrome c, mPTP opening, mitochondrial calcium release, oxygen consumption, mitochondrial membrane potential, ADP/ATP ratio, ATP concentration, and mitochondrial morphology. We found that IPF fibroblasts were resistant to mitomycin C-induced apoptosis and that calcium, a well-established activator of $\mathrm{mPTP}$, is decreased as well as the release of pro-apoptotic proteins such as cytochrome c. Likewise, IPF fibroblasts showed decreased mitochondrial function, while $\mathrm{MPTP}$ was less sensitive to ionomycin-induced opening. Although IPF fibroblasts did not present changes in the mitochondrial membrane potential, we found a fragmented mitochondrial network with scarce, thinned, and disordered mitochondria with reduced ATP levels. Our findings demonstrate that IPF fibroblasts are resistant to mitomycin C-induced apoptosis and that altered mPTP opening contributes to this resistance. In addition, IPF fibroblasts show mitochondrial dysfunction evidenced by a decrease in respiratory parameters.
\end{abstract}

Keywords: electron transport chain; IPF; mitochondrial dysfunction; mitochondrial networks; myofibroblasts 


\section{Introduction}

Idiopathic pulmonary fibrosis (IPF) is an aging-associated, progressive, and irreversible disease of unknown etiology. Currently, therapeutic options remain limited, and there is no efficient treatment to improve expectancy and quality of life $[1,2]$. Furthermore, the pathogenic mechanisms have not been fully elucidated. However, strong evidence suggests that IPF begins with micro-lesions and activation of the alveolar epithelium, which secretes various mediators that induce migration, proliferation, and activation of fibroblasts that produce excessive extracellular matrix amounts with progressive destruction of the lung parenchyma [3,4]. An intriguing aspect of the pathogenesis of IPF is the resistance shown by fibroblasts and myofibroblasts to apoptosis, while on the contrary, alveolar epithelial cells are very susceptible to this process [3-5]. The mechanisms of this paradoxical behavior are uncertain, but in the case of epithelial cells, their susceptibility has been attributed, at least partially, to mitochondrial dysfunction [5].

Fibroblasts and myofibroblasts are the critical effector cells in tissue fibrosis due to their participation in elaborating the extracellular matrix and contraction mechanisms and remodeling of damaged tissue [6]. During physiological regeneration, fibroblasts are eliminated by apoptosis, and evasion or resistance to this process has been associated with progressive fibrosis. In this context, it has been reported that fibroblasts and myofibroblasts from patients with IPF are resistant to apoptosis, although the molecular mechanisms involved are uncertain [7-10]. Due to the central role that mitochondria play in the implementation, amplification, and regulation of cellular apoptosis, this organelle would be involved in the apoptosis resistance of IPF fibroblasts, but this has not yet been studied in depth.

Mitochondria are central organelles that participate in vital metabolic processes, synthesize most ATP, and regulate several signaling cascades, including apoptosis [11]. The transduction of apoptotic signaling of cell death requires permeabilization of the mitochondrial membrane and the subsequent release of pro-apoptotic factors from the mitochondrial inter-membrane space (IMS) (i.e., cytochrome c, apoptosis-binding protein with low pI (Smac/Diablo), Endonuclease G, Serine protease Htra2 (Htra2/Omi), and apoptosisinducing factor (AIF)) [12]. The release of cytochrome $\mathrm{c}$ from mitochondria is commonly considered as the "point-of-no-return" in the sequence of events leading to apoptosis and involves changes in the mitochondrial membrane permeability transition (mtMPT) [13]. mtMPT results in the drop of the mitochondrial membrane potential $(\Delta \psi \mathrm{m})$, osmotic swelling of the mitochondrial matrix, rupture of the outer mitochondrial membrane, and release of cytochrome c [14].

The mitochondrial permeability transition pore (mPTP) is a non-specific voltagedependent channel that forms in the inner mitochondrial membrane. The molecular nature of MPTP is controversial due to the lack of knowledge about the identity of the proteins that form it. There are different hypotheses about the components that constitute the MPTP. One of them suggests the participation of the proteins adenine nucleotide translocator (ANT), voltage-dependent anion-selective channel (VDAC), and cyclophilin $\mathrm{D}$ (CypD), suggesting that ANT/VDAC form the basic unit of the mPTP, and that the recruitment of additional proteins, such as $\mathrm{CypD}$, Bax, hexokinase, or translocator protein (TSPO), modulate the activation of the MPTP. Another hypothesis recently developed has implicated different subunits of the $\mathrm{F}_{1} \mathrm{~F}_{0}$-ATP synthase as the inner membrane poreforming unit of the MPTP $[15,16]$.

Urbani et al. [17] proposed that active $\mathrm{F}_{1} \mathrm{~F}_{0}-\mathrm{ATP}$ synthase is responsible for the formation of $\mathrm{mPTP}$; they used highly purified $\mathrm{F}_{1} \mathrm{~F}_{0}$-ATP synthase and showed that calcium treatment mimics the response with specific agonists and inhibitors of mPTP. Recently, Pinke and Sazanov [18] also stated that the entire mammalian ATP synthase is part of the mPTP, based on an atomic model of ATP opening developed through cryogenic electron microscopy (cryo-EM) data. Despite these findings, the formation of the mPTP from $\mathrm{F}_{1} \mathrm{~F}_{0}$ ATP synthase has been questioned in recent studies, where some authors showed that $\mathrm{mPTP}$ persists in the absence of several subunits of $\mathrm{F}_{1} \mathrm{~F}_{0}$-ATP synthase. Since deleting such 
subunits prevents the assembly of functional $\mathrm{F}_{1} \mathrm{~F}_{0}$-ATP synthase, the authors concluded that ATP synthase does not participate directly in the mPTP formation $[19,20]$.

mPTP opening is triggered by matrix $\mathrm{Ca}^{2+}$, but its activity can be modulated by several other factors such as oxidative stress, adenine nucleotide depletion, high inorganic phosphate $(\mathrm{Pi})$ concentrations, mitochondrial membrane depolarization, or uncoupling [21]. The prolonged MPTP opening leads to an abrupt increase in the permeability of the inner mitochondrial membrane to solutes with molecular mass up to $1.5 \mathrm{kDa}$, provoking mitochondrial depolarization, followed by respiratory inhibition and the generation of reactive oxygen species (ROS), and massive release of matrix $\mathrm{Ca}^{2+}$.

This event induces the swelling of mitochondria, leading to breaks in the outer mitochondrial membrane that induce the release of pro-apoptotic factors [22]. For this reason, the mPTP has been proposed as a pivotal effector in the process of cell death [23,24].

Based on this accumulating evidence, we hypothesized that the resistance of IPF fibroblasts to apoptosis might be associated with a dysregulation in the mitochondrial function, mainly by resistance to the $\mathrm{MPTP}$ opening. Therefore, we aimed to analyze the mitochondrial function and the mPTP opening on the apoptosis resistance of human IPF fibroblasts. In the present study, we demonstrate that in IPF fibroblasts, mitochondria are dysmorphic and dysfunctional and that the resistance to apoptosis is related to altered mPTP opening. These findings may provide novel insights into the mechanisms involved in the resistance to apoptosis by IPF fibroblasts.

\section{Results}

\subsection{IPF Fibroblasts Are Resistant to Mitomycin C-Induced Apoptosis}

Mitomycin $C$ has been shown to inhibit fibroblast proliferation and to induce apoptosis through activation of the intrinsic mitochondrial pathway in human fibroblasts when administered in high doses $[25,26]$. To investigate the effect of mitomycin C on fibroblasts, we evaluated cell viability using the WST-1 assay following exposure to various mitomycin C concentrations $(10,25$, or $50 \mu \mathrm{g} / \mathrm{mL})$ for different times $(4,8,16$, or $24 \mathrm{~h})$. As shown in Figure $1 \mathrm{~A}$, mitomycin $\mathrm{C}$ significantly reduced the viability of control fibroblasts in a dose- and time-dependent manner. Treatment of control fibroblasts with mitomycin $\mathrm{C}$ $25 \mu \mathrm{g} / \mathrm{mL}$ for $24 \mathrm{~h}$ led to a nearly $50 \%$ decrease in cell viability. In contrast, mitomycin C did not produce the same effect in IPF fibroblasts. The maximum percentage of cell death was reached with mitomycin $\mathrm{C} 10 \mu \mathrm{g} / \mathrm{mL}$ at $8 \mathrm{~h}$, and no further cell death was reached by increasing the incubation time or mitomycin $C$ concentration. Representative bright-field micrographs using $25 \mu \mathrm{g} / \mathrm{mL}$ mitomycin $\mathrm{C}$ are shown in Figure 1B. To quantitatively examine the effect of mitomycin C on apoptosis, Annexin V-FITC/PI double staining was measured via flow cytometry in IPF and control fibroblasts. Following treatment with mitomycin C $(10,25$, and $50 \mu \mathrm{g} / \mathrm{mL}$ at $24 \mathrm{~h}$ ), the percentage of apoptotic cells (including early and late apoptosis) was evaluated. The results showed that IPF fibroblasts are significantly resistant to apoptosis induced by mitomycin $C$ at 25 and $50 \mu \mathrm{g} / \mathrm{mL}$, compared with control fibroblasts $\left(* * p<0.05\right.$ or ${ }^{* * *} p<0.01$ ) Figure $1 \mathrm{C}$.

\subsection{IPF Fibroblasts Show a Decrease in the Mitomycin C-Induced Release of Cytochrome c}

To determine whether the resistance to apoptosis in IPF fibroblasts is associated with the release of cytochrome c, IPF and control fibroblasts were stimulated with mitomycin C $(25 \mu \mathrm{g} / \mathrm{mL})$ for 4 and $24 \mathrm{~h}$. Subsequently, the cytosolic and mitochondrial fractions were isolated. The release of cytochrome $\mathrm{c}$ was determined in the cytosolic fraction by Western blot (WB) and HPLC, while the mitochondrial fraction was only analyzed by WB due to the small amount of sample obtained during isolation. As illustrated in Figure 2A, the cytosolic fraction isolated from control fibroblasts had a higher cytochrome c content after mitomycin $\mathrm{C}$ treatment than basal condition (*** $p<0.01$ ). In contrast, the cytosolic cytochrome $\mathrm{c}$ content did not change in IPF fibroblasts after mitomycin $C$ treatment. These results were confirmed by HPLC analysis (Figure 2B). Additionally, we analyzed the expression levels of Bax, a Bcl-2-associated X protein. We found a strong decrease of Bax in the cytosolic 
fraction of control fibroblasts stimulated with mitomycin $C$ for $24 \mathrm{~h}$, suggesting that Bax was translocated from the cytosol to the mitochondrial membrane, contributing to the apoptosis process. By contrast, the level of this protein showed only a slight decrease at $24 \mathrm{~h}$ in IPF fibroblasts. Moreover, the stimulation with mitomycin $\mathrm{C}$ for $24 \mathrm{~h}$ increased the active form of caspase 9 in the cytosolic fraction of control fibroblasts $\left({ }^{*} p<0.05\right)$ but not in IPF fibroblasts (Figure 2A). These results show that the intrinsic pathway is activated in control fibroblasts but not in IPF fibroblasts.

A)

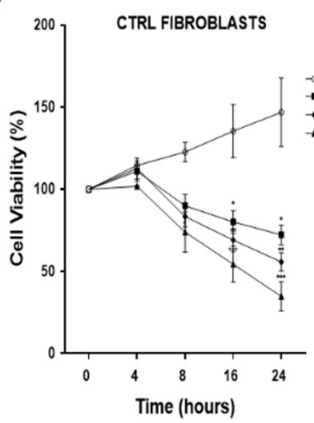

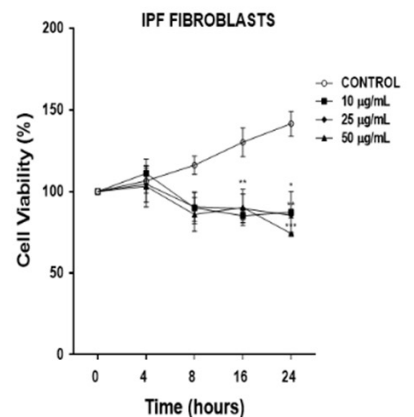

B)

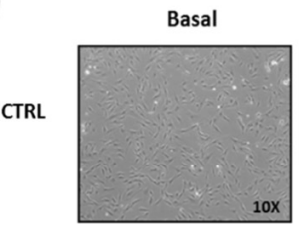

Basal

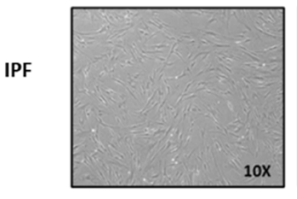

MMC $4 \mathbf{h}$

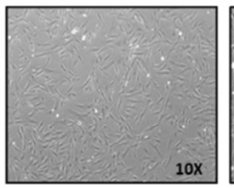

MMC $4 \mathrm{~h}$

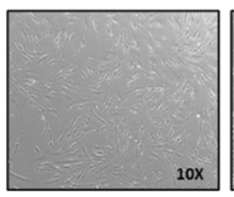

MMC $24 \mathrm{~h}$

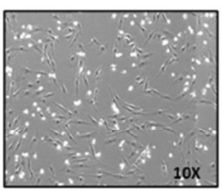

MMC $24 \mathrm{~h}$

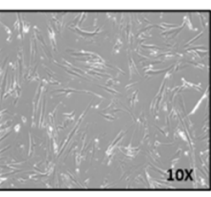

c)
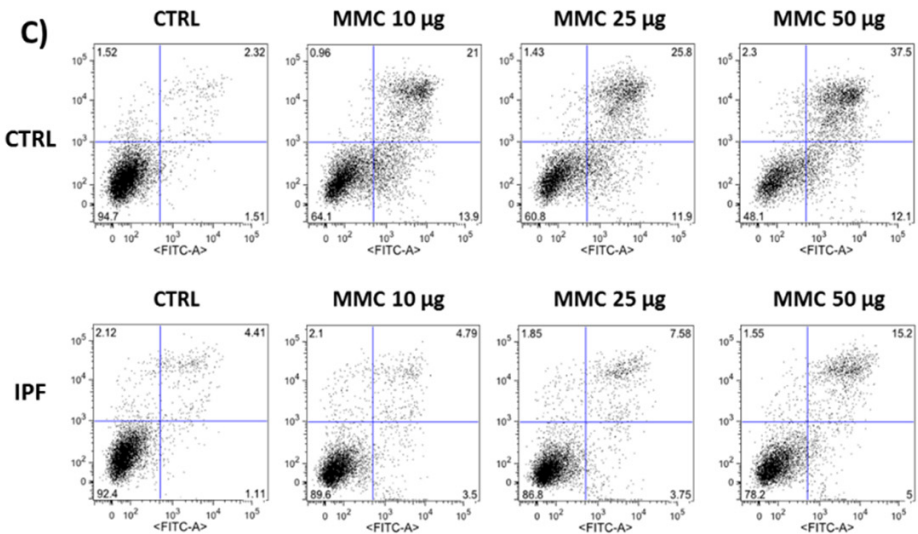

Figure 1. Effect of mitomycin $C$ (MMC) on viability and resistance to apoptosis in idiopathic pulmonary fibrosis and normal lung fibroblasts. (A) After treatment with increasing concentration of mitomycin C for 4, 8, 16, and $24 \mathrm{~h}$, the effect on cell viability was determined using the WST- 1 assay. The data represent the means \pm SEM of two independent experiments ${ }^{* *} p<0.01,{ }^{* * *} p<0.01$, compared with the control group. (B) Bright field representative images from IPF and control fibroblasts after incubation with mitomycin C $(25 \mu \mathrm{g} / \mathrm{mL})$ for 4 and $24 \mathrm{~h}$. (C) Cell apoptosis rates were analyzed via flow cytometry using the Annexin V/propidium iodide (FITC/PI) double staining method. Fibroblasts were stimulated with mitomycin $\mathrm{C}$ for $24 \mathrm{~h}$. Staurosporine (STS, $1 \mu \mathrm{M}$ ) was used as a positive control. The results are expressed as the mean \pm SEM. The differences were analyzed using two-way ANOVA followed by the Bonferroni multiple comparisons test. ${ }^{* *} p<0.05$ or ${ }^{* * *} p<0.01$ vs. control. CTRL $(n=2)$ and IPF $(n=4)$.

On the other hand, the analysis of the mitochondrial fraction of control fibroblasts showed a high content of cytochrome $\mathrm{c}$ under basal conditions, while the mitomycin $\mathrm{C}$ treatment for $24 \mathrm{~h}$ provoked a significant decrease $(* * *<0.01)$. Interestingly, under basal conditions, the mitochondrial cytochrome c content in IPF fibroblasts was significantly lower than in control fibroblasts, while treatment did not change their levels (Figure 2C). As a whole, these data indicate that IPF fibroblasts released fewer cytochrome c after stimulation with $25 \mu \mathrm{g} / \mathrm{mL}$ mitomycin $\mathrm{C}$ at $24 \mathrm{~h}$ compared with control fibroblasts. 
A)

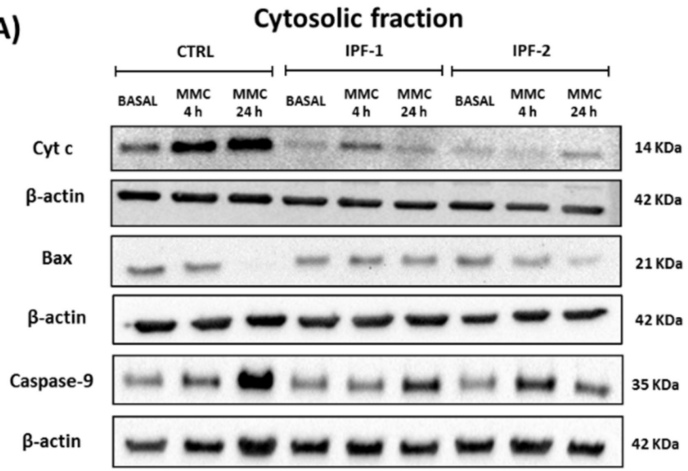

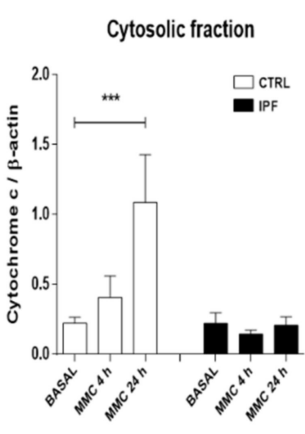

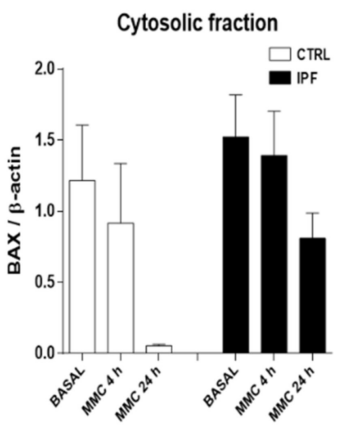

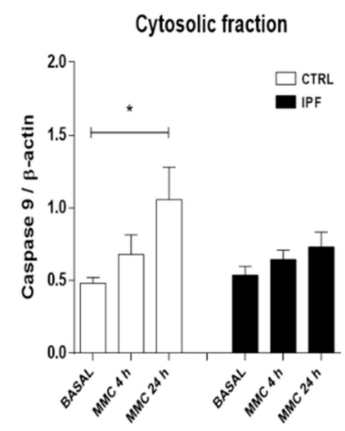

B)
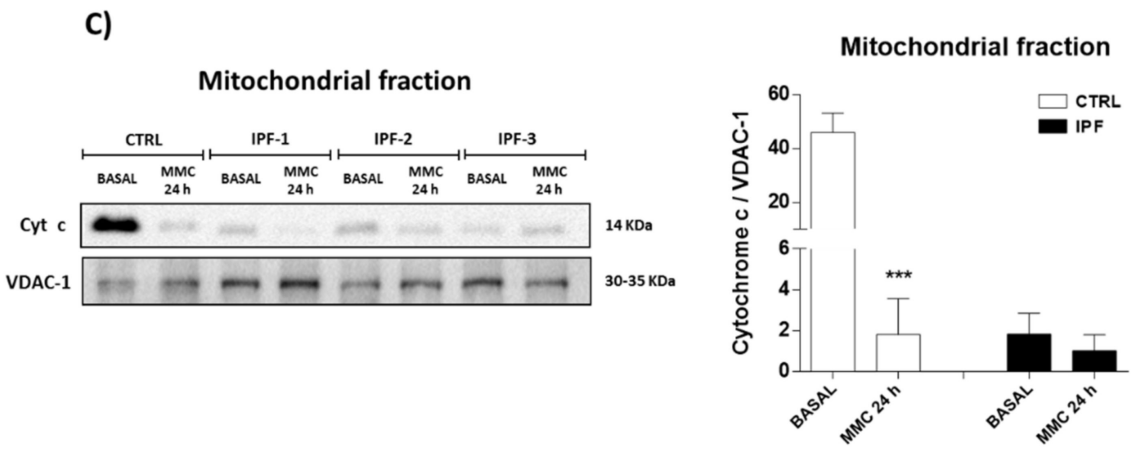

Figure 2. Fibroblasts from idiopathic pulmonary fibrosis show a decrease in the release of cytochrome c induced for mitomycin C (MMC). After exposure to $25 \mu \mathrm{g} / \mathrm{mL}$ mitomycin $\mathrm{C}$ for 4 and $24 \mathrm{~h}$, we isolated the cytosolic and mitochondrial fractions in control and IPF fibroblasts. (A) Expression levels of cytochrome c, caspase 9, and Bax in cytosolic fraction were determined via Western blot analysis. The expression levels of $\beta$-actin were used as a loading control. (B) Cytosolic fraction was also analyzed by the high-performance liquid chromatography (HPLC) technique. (C) The expression levels of cytochrome $\mathrm{c}$ in mitochondrial fraction were determined via Western blot analysis. Expression levels of VDAC-1 were used as a loading control. The results are expressed as the mean \pm SEM of at least three independent experiments. The differences were analyzed using one-way ANOVA followed by Dunnett's multiple comparisons test, ${ }^{*} p<0.05,{ }^{* * *} p<0.01$ vs. control, CTRL $(n=3)$ and $\operatorname{IPF}(n=3)$.

\subsection{IPF Fibroblasts Show Strong Resistance to mPTP Opening Induced By Ionomycin}

It has been proposed that modulation of MPTP opening is a strategic regulator of cell death by apoptosis. We determined whether mPTP opening was associated with the apoptosis resistance shown by IPF fibroblasts. We compared the opening of mPTP in control and IPF fibroblasts under basal conditions using the $\mathrm{CoCl}_{2}$-calcein fluorescence-quenching assay. First, we measured the calcein fluorescence by confocal microscopy in the absence of cobalt. As expected, calcein fluorescence is much higher in the absence of $\mathrm{CoCl}_{2}\left(\mathrm{Co}^{2+}\right.$-free). The results showed similar levels of calcein between IPF and normal lung fibroblasts, indicating similar calcein loading (Figure 3A,B). However, in the presence of $\mathrm{CoCl}_{2}$, calcein fluorescence inside mitochondria is lower in control than in IPF fibroblasts $\left({ }^{*} p<0.05\right)$. This result suggests that $\mathrm{mPTP}$ is more open in controls than IPF fibroblasts (Figure 3A,B). We also analyzed mPTP opening in fibroblasts by flow cytometry using a MitoProbe Transition Pore Assay Kit to corroborate this finding. The change in fluorescence between cells incubated with only $\mathrm{CoCl}_{2}$ and those incubated with ionomycin indicates the continuous activation of MPTP. Our results showed that MPTP opening induced by ionomycin was higher in control than in IPF fibroblasts ( ${ }^{* * *} p<0.01$ vs. control) (Figure $\left.3 C, D\right)$. This result confirmed that IPF fibroblasts are most resistant to mPTP opening ionomycin-induced, as demonstrated by the retention of calcein fluorescence in the presence of ionomycin in IPF fibroblasts (Figure 4A). We also examined the expression levels of ANT-1 protein, and as shown in Figure 4B, these were markedly lower in IPF compared with controls 
fibroblasts ( ${ }^{*} p<0.05$ vs. control). This result is captivating because this protein participates in mPTP formation.

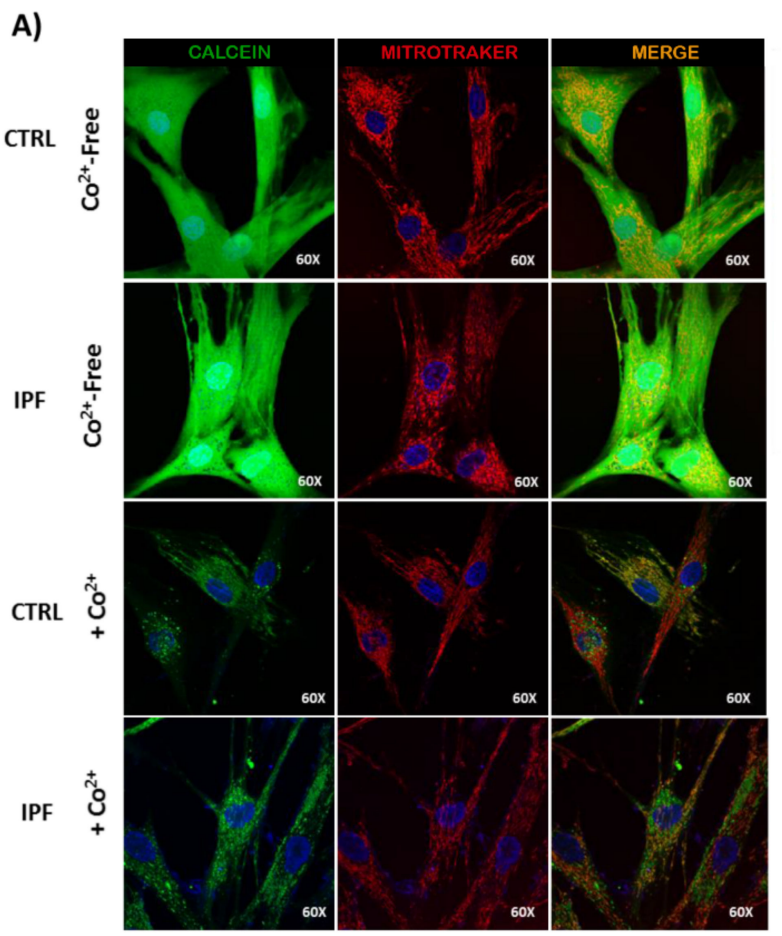

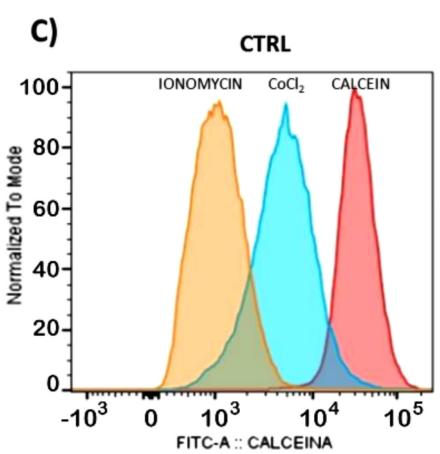

B)

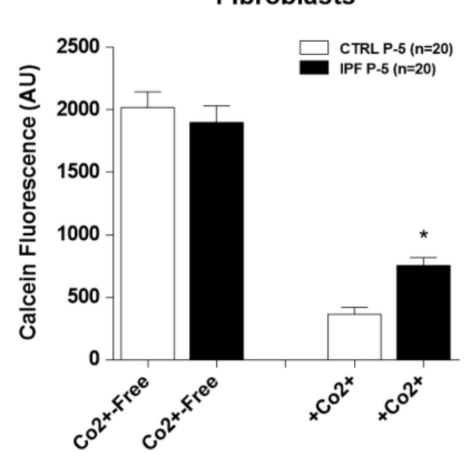

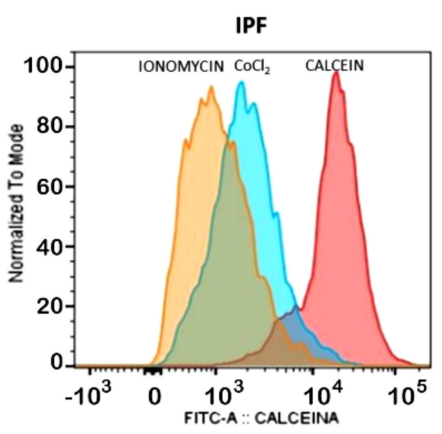

D)

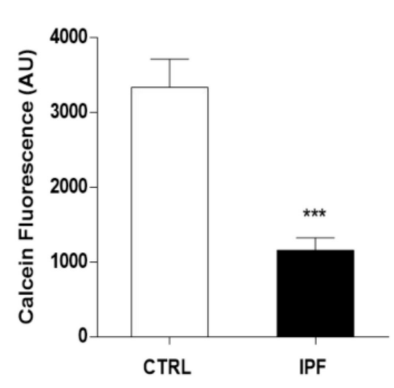

Figure 3. Idiopathic pulmonary fibrosis fibroblasts show strong resistance to ionomycin-induced mPTP opening. (A) Representative confocal micrographs of IPF and control fibroblasts after incubation with calcein-AM $(1 \mu \mathrm{M})$ and MitoTracker Red $(150 \mathrm{nM})$ in the presence or absence of $\mathrm{Co}^{2+}(1 \mathrm{mM})$, which quenches calcein fluorescence (green) outside of mitochond ria. In the absence of $\mathrm{Co}^{2+}$, the calcein fluorescent signal is very intense and is present in the entire cell. The calcein fluorescence in mitochondria is lower in the presence of $\mathrm{Co}^{2+}$. (B) The bar graph shows the quantification of calcein fluorescence in IPF and control cells in the absence or presence of $\mathrm{Co}^{2+}$. The number shown in the panel indicates the number of cells used in the study. The results are expressed as the mean \pm SEM. The differences were analyzed using one-way ANOVA followed by the Bonferroni multiple comparisons test. ${ }^{*} p<0.05$. (C) Representative flow cytometry histogram shows the intensity of calcein signals in IPF and control fibroblasts. The change in fluorescence between $\mathrm{Co}^{2+}$ and ionomycin indicates the continuous activation of mitochondrial permeability transition pores. (D) The bar graph shows the quantification of mean calcein fluorescence in IPF and control cells. The results are expressed as the mean $\pm \mathrm{SEM}$. The differences were analyzed using an unpaired $t$-test. ${ }^{* * *} p<0.01$ vs. control. CTRL $(n=3)$ and IPF $(n=4)$.

\subsection{IPF Fibroblasts Show Decreased Release Mitochondrial Calcium}

We also evaluated mitochondrial calcium in control and IPF fibroblasts, since elevated mitochondrial calcium levels induces the opening of the mPTP. We used FCCP to increases the $\left[\mathrm{Ca}^{2+}\right]_{i}$ by activating the mPTP in control and IPF fibroblasts. Our results indicate that FCCP elicited in normal fibroblasts a transient moderate increase in $\left[\mathrm{Ca}^{2+}\right]_{\mathrm{i}}$ to $115.76 \pm 9.53$ and $108.09 \pm 7.01 \mathrm{nM}$, respectively (Figure 5A). In contrast, the IPF fibroblasts exhibited a significantly diminished increase in $\left[\mathrm{Ca}^{2+}\right]_{\mathrm{i}}$ levels by $13.59 \pm 1.49$ and $18.36 \pm 5.24 \mathrm{nM}$, respectively (Figure $5 \mathrm{~B}, \mathrm{C})\left({ }^{* * *} p<0.01\right)$. To corroborate that the increase in $\left[\mathrm{Ca}^{2+}\right]_{\mathrm{i}}$ is due to the activation of the MPTP with FCCP, we blocked pharmacologically the mPTP with cyclosporin A, inhibiting the response (Figure 5D). 
A)
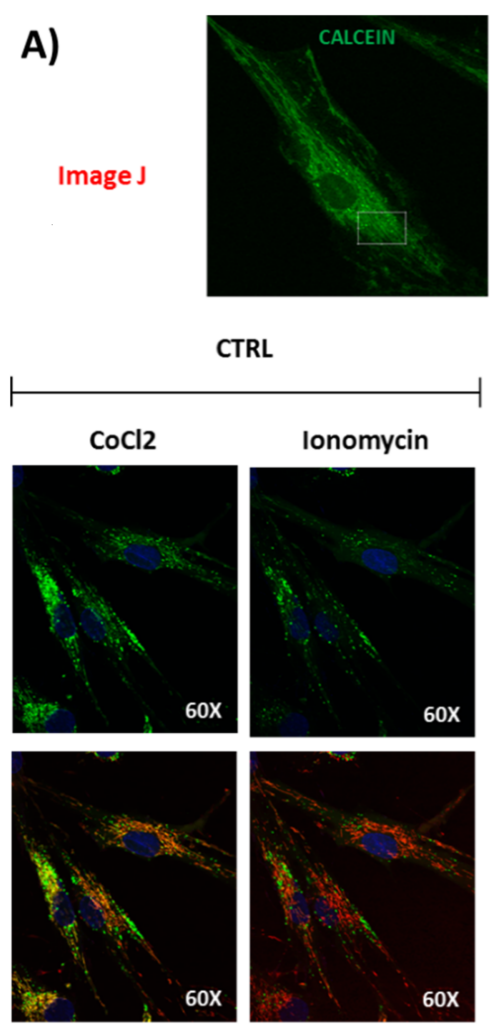

TRL

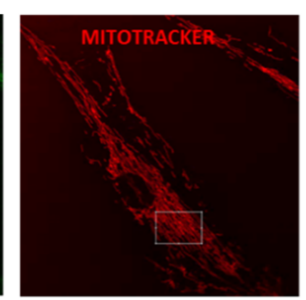

IPF

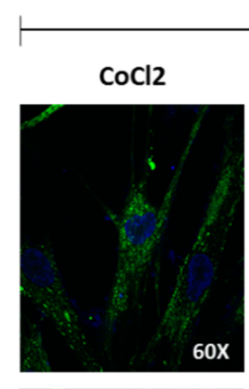

$60 \mathrm{x}$

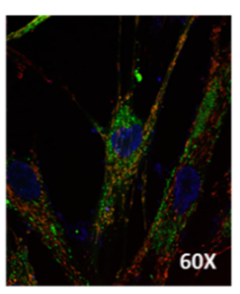

B)

Adenine Nucleotide Translocator (ANT)
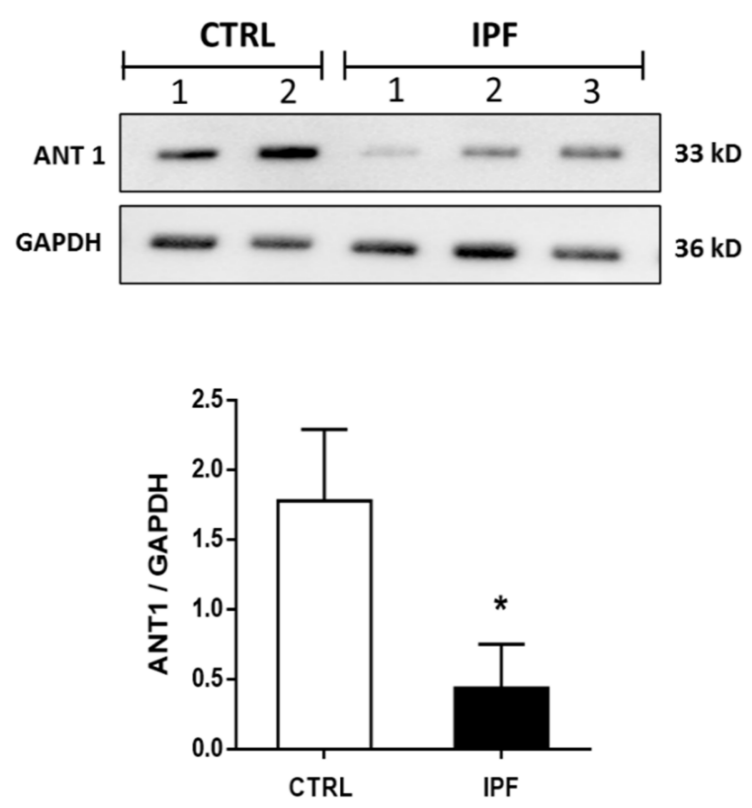

Figure 4. Idiopathic pulmonary fibrosis fibroblasts show resistance to mPTP opening induced by ionomycin. (A) Representative confocal micrographs of IPF and control fibroblasts after incubation with calcein-AM (1 $\mu$ M), MitoTracker Red $(150 \mathrm{nM})$, and $\mathrm{Co}^{2+}(1 \mathrm{mM})$, in the presence or absence of ionomycin $(1 \mu \mathrm{M})$, which quenches calcein fluorescence (green) within mitochondria. The fluorescence of calcein is maintained in IPF fibroblasts after ionomycin administration compared to control fibroblasts. (B) Image representative of ANT protein expression in control and IPF fibroblasts. The expression levels of GAPDH were used as a loading control. The results are expressed as the mean \pm SEM. The differences were analyzed using an unpaired $t$-test. ${ }^{*} p<0.05$ vs. control, CTRL $(n=2)$ and IPF $(n=3)$, one experiment.

\subsection{Inhibition of the MPTP Opening with BKA Decreased Apoptosis in Control Fibroblasts}

To determine whether the inhibition of mPTP opening participates in the resistance of fibroblasts to apoptosis, control fibroblasts were stimulated with BKA (10 $\mu \mathrm{M}$, for $6 \mathrm{~h})$, an inhibitor of the opening of $\mathrm{mPTP}$, and then were stimulated with mitomycin $\mathrm{C}(25 \mu \mathrm{g} / \mathrm{mL})$ for $24 \mathrm{~h}$. Our results showed an increase in the number of positive apoptotic cells when we used mitomycin C. Interestingly, the administration of BKA decreased the percentage of apoptotic cells in control fibroblasts $\left.{ }^{* *} p<0.01\right)$, which are very susceptible to cell death (Figure 6A). On the other hand, when we stimulated control fibroblasts with ATR, an inducer of mPTP opening, we found a slight and not significant increase in the percentage of apoptosis induced by mitomycin C (Figure 6B).

\subsection{IPF Fibroblasts Exhibit Lower Oxygen Consumption Than Control Fibroblasts}

To investigate whether apoptosis resistance in IPF fibroblasts is associated with mitochondrial dysfunction, we analyzed the integrity of mitochondria through oxygen consumption in IPF and control fibroblasts. Oxygen flux ( $\mathrm{pmol} \mathrm{O} 2 / \mathrm{s} / 10^{6}$ cells $/ \mathrm{mL}$ ) was determined in $2 \times 10^{6}$ non-permeabilized fibroblasts. Figure 7A shows a representative trace of control and IPF fibroblasts respiration. The routine respiration of fibroblasts was measured in standard incubation media (10-20 min), and then, we performed sequential injections of substrate-uncoupler-inhibitor-titration for key parameters of mitochondrial respiration. As shown in Figure 7B, routine respiration of control fibroblasts was higher than IPF fibroblasts $(* * p<0.01)$, which was suppressed by oligomycin, a specific inhibitor of mitochondrial ATP synthase and oxidative phosphorylation. The proton leak 
was slightly increased in the IPF fibroblasts, but the difference against control fibroblasts was not significant $(p=0.06)$. The maximal respiration of fibroblasts was determined after the uncoupling of mitochondria with CCCP. The results showed that IPF fibroblasts had a decreased maximal respiration compared with the control group $\left({ }^{* *} p<0.01\right)$. Finally, respiratory control (RC) was calculated by the difference between basal respiration and proton leak. The results showed that IPF fibroblasts exhibited lower RC than normal fibroblasts (*** $p<0.01)$. Together, our findings indicate impaired respiration in IPF fibroblasts. We evaluated the oxygen consumption in permeabilized fibroblasts to determine whether the modification of respiration in IPF fibroblasts was associated with an alteration in complex I and II of the electron transport chain. We determined the electron transport chain activity using specific substrates to complex I (glutamate/malate) and complex II (succinate). A representative trace is shown in Figure $7 \mathrm{C}$. The electron transport chain activity was decreased in IPF fibroblasts when it was stimulated through complex I $(* * p<0.01)$ or complex II $\left({ }^{*} p<0.05\right)$ (Figure 7D).

A)

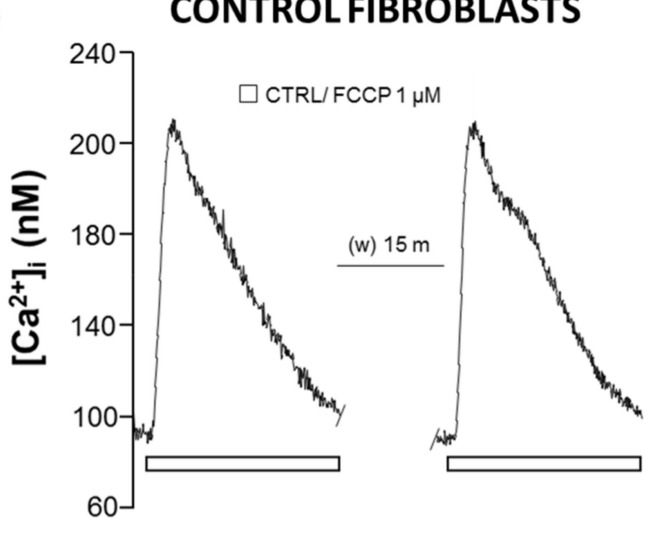

C)

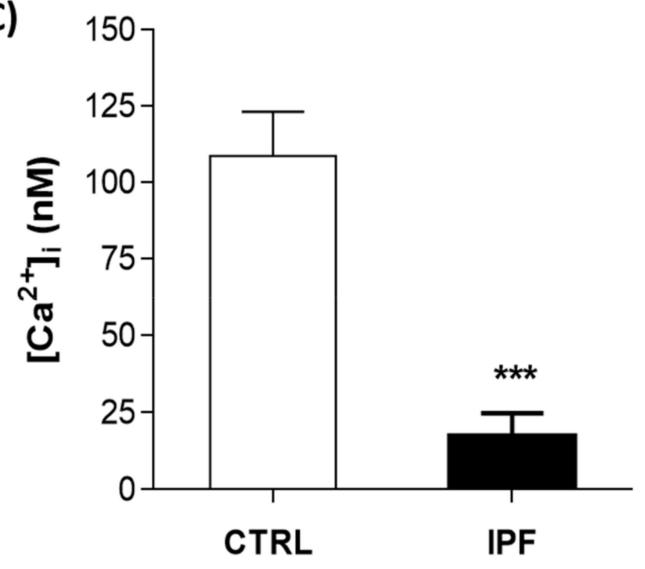

B)

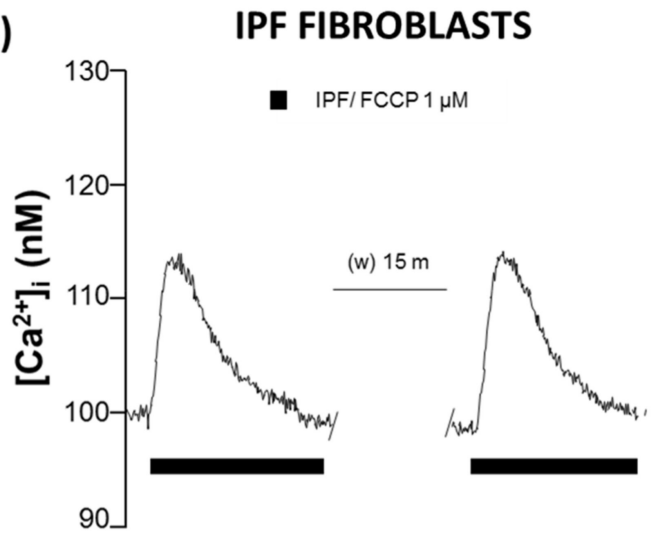

D)

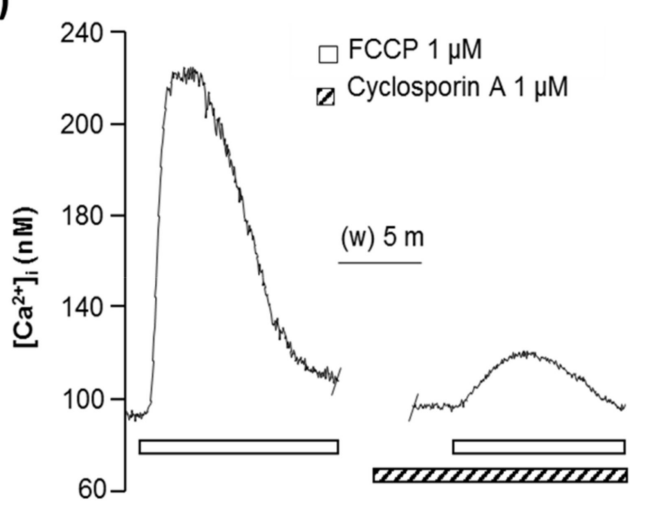

Figure 5. Typical recordings of simultaneous intracellular calcium concentration assay ([Ca $\left.\left.{ }^{2+}\right] \mathrm{i}\right)$ control fibroblasts and IPF. Two pulses of FCPP were applied with an interstimulus interval of $15 \mathrm{~min}$ to determine the response reproducibility (A,B respectively) (CTRL $n=2$; IPF $n=2$ ). Six fibroblasts were evaluated for each experimental condition. (C) Data are shown as media \pm SEM and were compared by Student's $t$-test, ${ }^{* * *} p<0.01$. (D) $\mathrm{Ca}^{2+}$ increase induced by FCCP was blocked by cyclosporin A $(n=2)$. 
A)

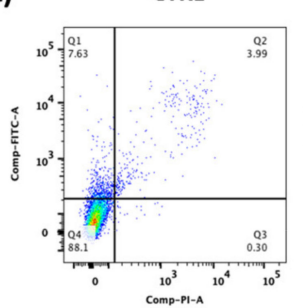

B)

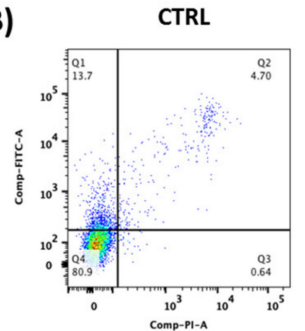

MMC $(25 \mu \mathrm{M})$

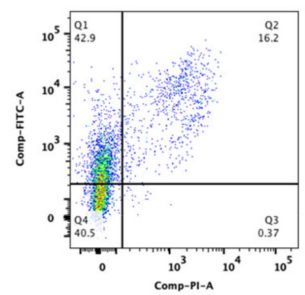

MMC $(25 \mu \mathrm{M})$

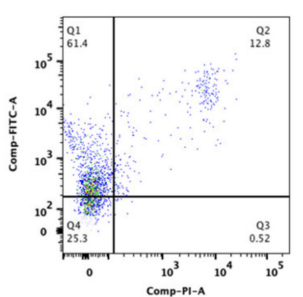

BKA $(10 \mu \mathrm{M})$
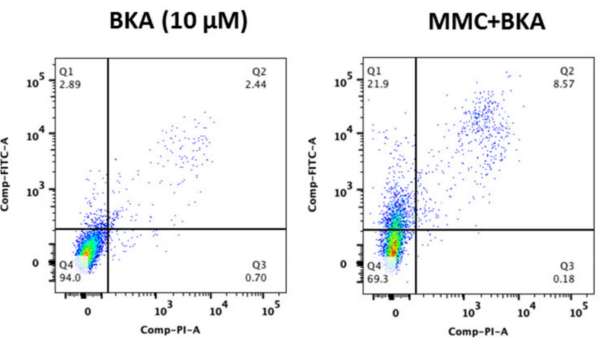

MMC+ATR
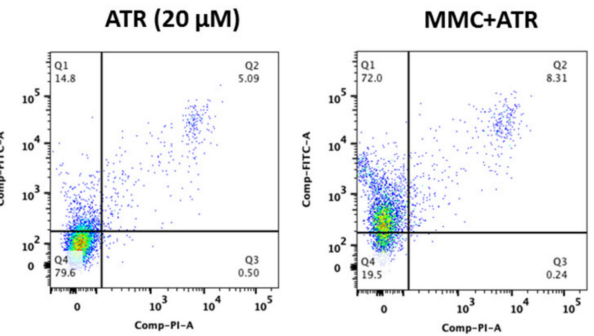
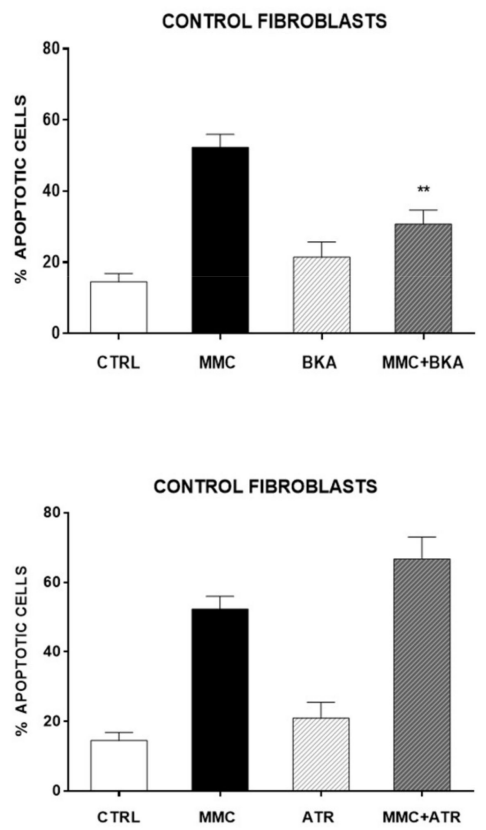

Figure 6. Control fibroblasts show resistance to apoptosis induced by mitomycin $C$ in the presence of bongkrekic acid (BKA) without changes with atractyloside (ATR). (A) Representative image of dot plot analysis by flow cytometry. Cell apoptosis rates were analyzed using the Annexin V-FITC/PI double-staining method. (B) Fibroblasts were stimulated with BKA $(10 \mu \mathrm{M})$ for $6 \mathrm{~h}$, and then, we incubated fibroblasts with mitomycin C MMC $(25 \mu \mathrm{g} / \mathrm{mL})$ for $24 \mathrm{~h}$. Fibroblasts were stimulated with ATR $(20 \mu \mathrm{M})$ for $1 \mathrm{~h}$, and then, we incubated fibroblasts with $(25 \mu \mathrm{g} / \mathrm{mL})$ for $24 \mathrm{~h}$. The results are expressed as the mean \pm SEM. The differences were analyzed using one-way ANOVA followed by the Bonferroni multiple comparisons test, ${ }^{* *} p<0.01$ vs. control. CTRL $(n=3)$.

\subsection{The Mitochondrial Transmembrane Potential Is Similar in IPF and Control Fibroblasts}

As we detected alterations in oxygen consumption, we turned our attention toward the mitochondrial membrane potential: the electrochemical force that modulates the kinetics of proton re-entry to the matrix through ATP synthase. Using confocal microscopy, we measured the $\Delta \Psi \mathrm{m}$ of control and IPF fibroblast stained with JC-1 $(4 \mu \mathrm{M})$. JC-1 is a cationic fluorescent dye that exhibits potential-dependent accumulation in mitochondria (Figure 8A). We did not find significant differences in the JC-1 fluorescence signal between control and IPF fibroblasts (Figure $8 \mathrm{~B}$ ). To ensure that dye is equally loaded and that the JC-1 signal is not auto-quenched, we compared JC-1 fluorescence in control and IPF fibroblasts following oligomycin or FCCP treatment. Oligomycin, an inhibitor of ATP synthase, induces hyperpolarization of mitochondria and increases JC-1 fluorescence, whereas FCCP dissipates transmembrane potential (Figure 8B).

\subsection{IPF Fibroblasts Display Decreased ATP Levels but Do Not Show a Change in ADP/ATP Ratio}

Diverse studies have proposed the participation of ATP synthase in the formation of $\mathrm{mPTP}$. For this reason, we evaluated the levels of expression of ATP synthase by Western blot in control and IPF fibroblasts, and no differences were found (Figure 8C). Furthermore, we evaluated the ADP/ATP ratio in control and IPF fibroblasts, and also, no significant differences were observed, suggesting no changes in ATP synthase activity (Figure 8D).

ATP production in IPF and control fibroblasts was determined quantitatively through a luminescence assay. The results showed that IPF fibroblasts had a lower ATP total concentration than the control group $(* *<0.01)$. In addition, decreased mitochondrial respiration in IPF fibroblasts was correlated with a decrease in ATP production (Figure 8E). 
A)

CTRL

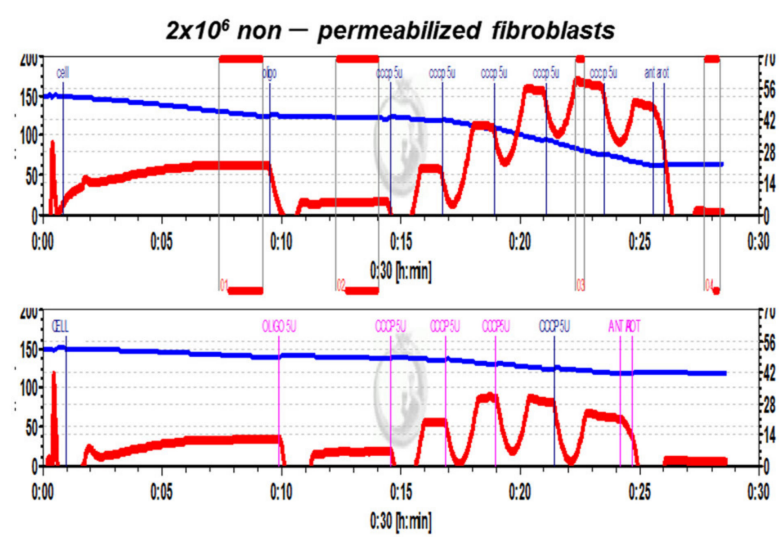

C)

$5 \times 10^{5}$ permeabilized fibroblasts with digitonin

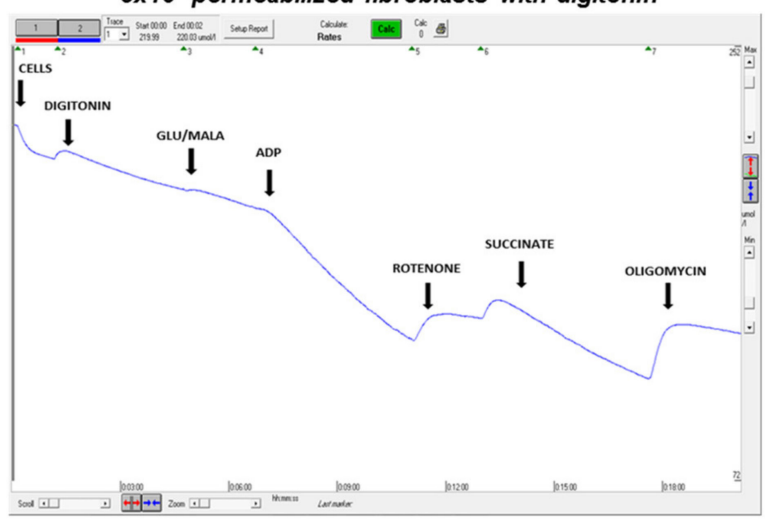

B)

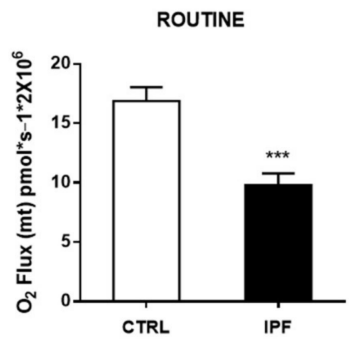

MAXIMAL RESPIRATION

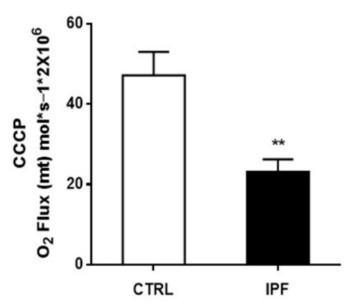

D)

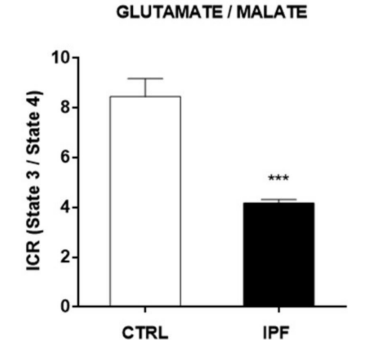

PROTON LEAK

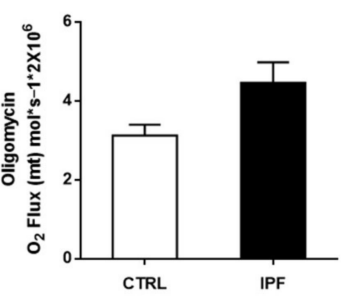

RESPIRATORY CONTROL

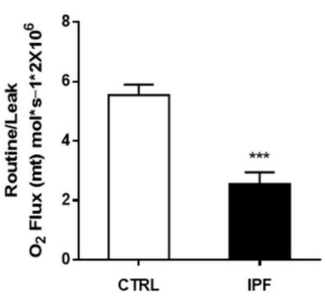

SUCCINATE

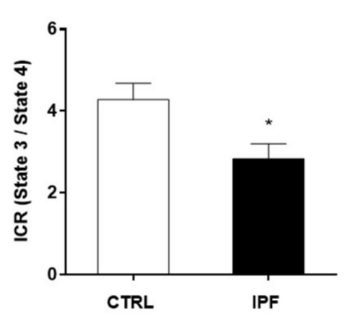

Figure 7. Oxygen consumption in idiopathic pulmonary fibrosis and control fibroblasts with and without induction of permeability. Intact fibroblasts were placed in a $2 \mathrm{~mL}$ chamber at a final concentration of $2 \times 10^{6}$ cells $/ \mathrm{mL}$. Measurement of oxygen consumption was performed at $37^{\circ} \mathrm{C}$ in high-resolution oxygraphy (Oxygraph-2k Oroboros Instruments, Innsbruck, Austria). Proton leak was induced by oligomycin $(5 \mu \mathrm{M})$, and titrations of $1 \mu \mathrm{M}$ CCCP induced the maximum uncoupled respiration. Finally, we inhibit the OXPHOS with rotenone and antimycin. (A) Representative traces of oxygen consumption in control and IPF fibroblasts. (B) Graphs of different parameters of mitochondrial respiration (Routine, Proton Leak, Maximal Respiration, and Respiratory Control). The results are expressed as the mean \pm SEM. The differences were analyzed using an unpaired $t$-test, ${ }^{* *} p<0.05,{ }^{* * *} p<0.01$ vs. control. CTRL $(n=2)$ and IPF $(n=4)$. (C) Representative traces of oxygen consumption using different substrates for complex I and II. (D) Graphs of respiratory control index (ICR) using glutamate/malate $(10 \mathrm{mM} / 5 \mathrm{mM})$ and succinate $(5 \mathrm{mM})$. Respiration inhibition was carried out in the presence of rotenone $(0.5 \mu \mathrm{M})$ by complex I and oligomycin $(5 \mu \mathrm{M})$ complex II. The results are expressed as the mean \pm SEM. The differences were analyzed using an unpaired $t$-test, ${ }^{*} p<0.05,{ }^{* *} p<0.01$ vs. control. CTRL $(n=3)$ and $\operatorname{IPF}(n=2)$.

\subsection{IPF Fibroblasts Display Abnormal Mitochondrial Structure}

In order to evaluate the mitochondrial structure, we analyzed IPF and control fibroblasts by fluorescence and transmission electron microscopy (TEM). As shown in the fluorescence micrograph with MitoTracker, we observed a mitochondrial network fragmented and accumulation of mitochondria in IPF compared to control fibroblasts. Moreover, fibroblasts from control lungs showed branched, elongated, and lengthwise organized mitochondria with a typical appearance; the cristae were electrodense and compact. In contrast, in IPF fibroblasts, the mitochondria were scarce, thinned, and disordered; the cristae showed a lesser electron density than control fibroblasts (Figure 9A). As shown in the frequency histogram, control fibroblasts mitochondria have a size of around $600 \mathrm{~nm}^{2}$, while mitochondria from patients with IPF have a much smaller size of about $250 \mathrm{~nm}^{2}$ (Figure 9B). We also evaluated the mitochondrial elongation by the length of mitochondria mean per image using the ImageJ software. The IPF mitochondria exhibited lower elongation than mitochondrial control (Figure 9C). 

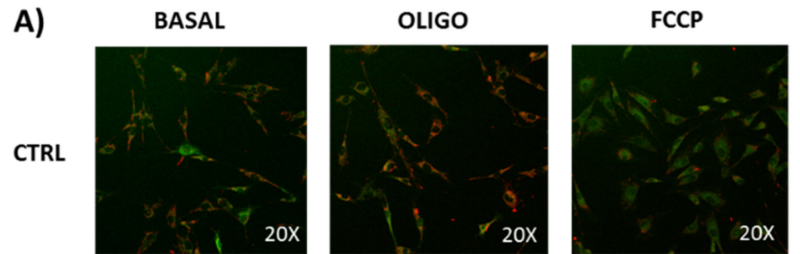

IPF
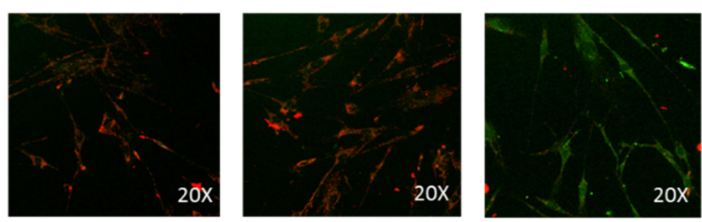

B)

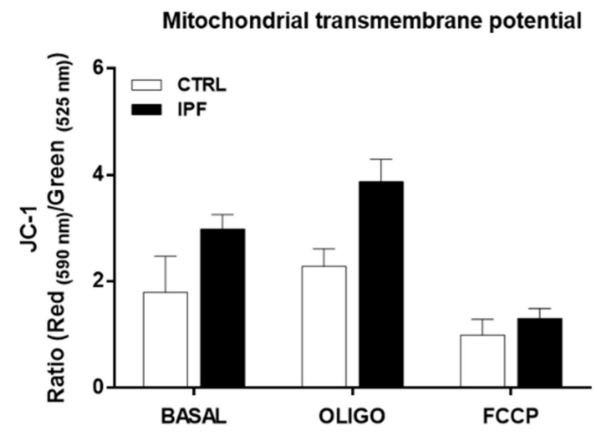

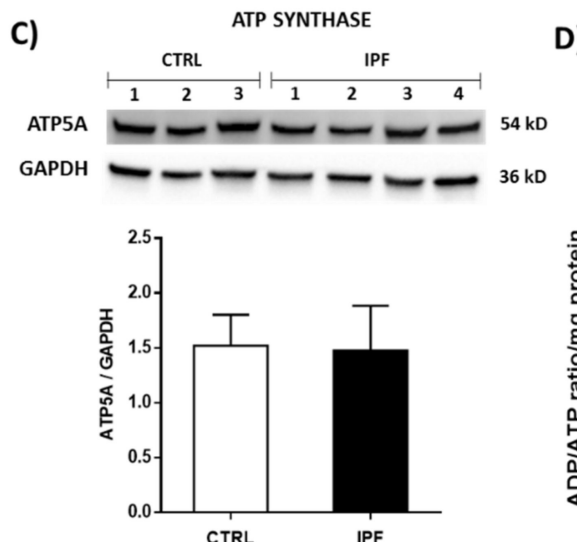

E)

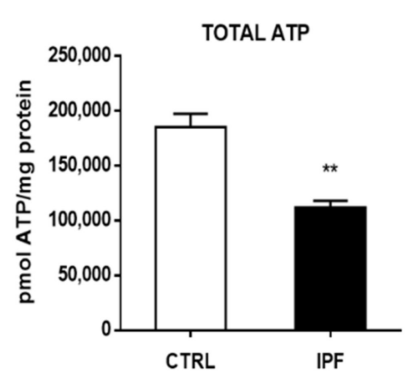

Figure 8. IPF fibroblasts do not modify the mitochondrial transmembrane potential or the ADP/ATP ratio but decrease the total levels of ATP. (A) Representative fluorescence microscopic image of control and IPF fibroblasts after staining with JC-1 $(4 \mu \mathrm{M})$ in the presence or absence of oligomycin $(1 \mu \mathrm{M})$ or FCCP $(10 \mu \mathrm{M})$. The intensity of JC-1 reflects the level of mitochondrial transmembrane potential. (B) The bar graph shows the quantification of JC-1 signals in control and IPF fibroblasts in the presence or absence of oligomycin and FCCP using fluorescence microscopy. The results are expressed as the mean \pm SEM. CTRL $(n=1)$ and IPF $(n=2)(C)$ Expression levels of ATP synthase in the lysate of control and IPF fibroblasts were determined via Western blot analysis. The expression levels of GAPDH were used as a loading control CTRL $(n=3)$ and IPF $(n=4)$ from three independent experiments. (D) ADP/ATP ratio was quantified by a luciferin/luciferase-based luminescence assay. The results are expressed as the mean $\pm \mathrm{SEM}$. The differences were analyzed using an unpaired $t$-test. CTRL $(n=4)$ and IPF $(n=4)$. (E) ATP production was quantified by luminescence assay in control and IPF fibroblasts. The results are expressed as the mean \pm SEM. The differences were analyzed using an unpaired $t$-test. ** $p<0.01$ vs. control. CTRL $(n=3)$ and $\operatorname{IPF}(n=3)$.

We also determined by confocal microscopy, through a Z-stack analysis, the mean fluorescence intensity of the MitoTracker in whole cells in control and IPF fibroblasts. In addition, sections were made throughout the entire cell to indirectly assess whether there were changes in mitochondrial mass. Our results show no significant change in the fluorescence of the mitochondrial marker MitoTracker between control and IPF fibroblasts, suggesting that there are no significant differences in the number of mitochondria (Figure 10). In addition, previous studies have shown that there are no differences in the amount of mitochondrial DNA between normal lung and IPF fibroblasts [5]. 

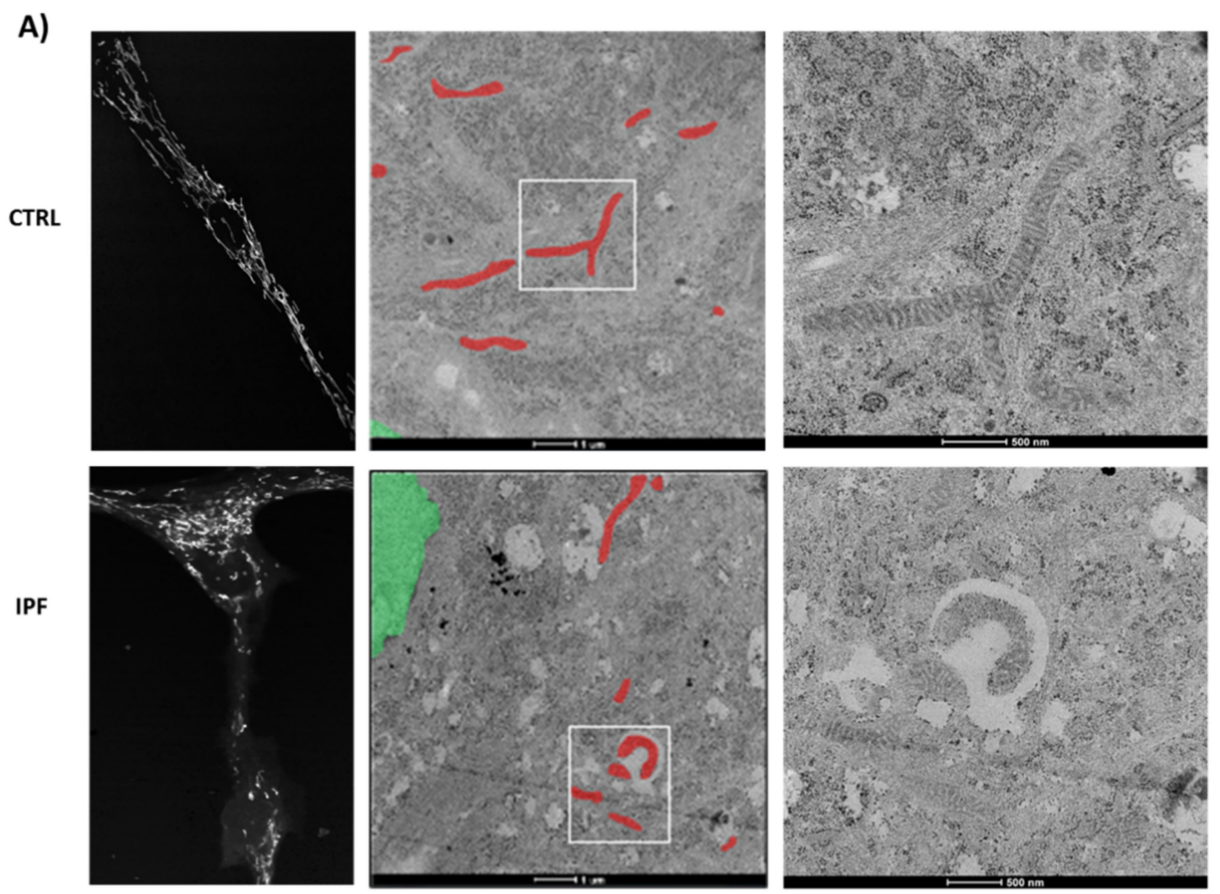

B)

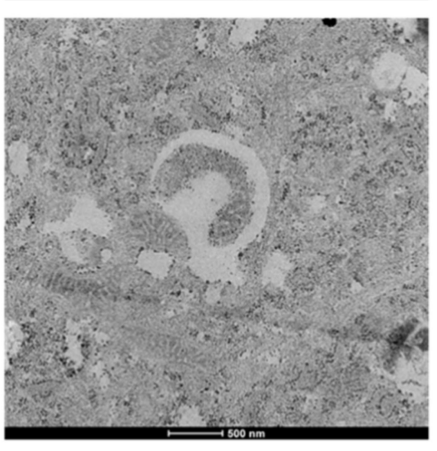

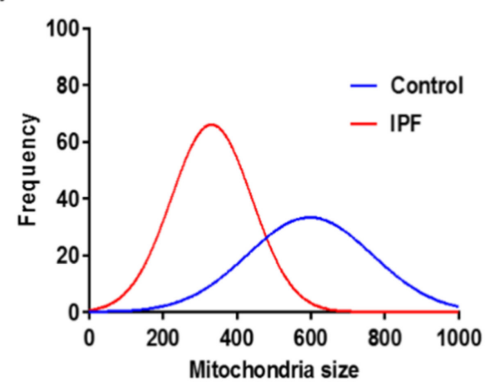

c)

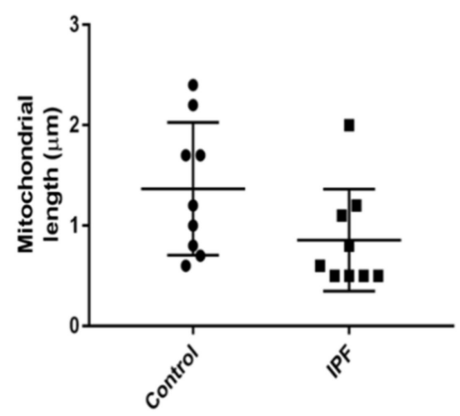

Figure 9. Number and morphology of lung fibroblasts mitochondria from healthy donors and idiopathic pulmonary fibrosis patients. (A) Representative confocal and transmission electron microscopy of mitochondrial morphology of lung fibroblasts derived from healthy donors and IPF patients. Two different magnifications of the same cell are shown, and mitochondria are highlighted in red. (B) Frequency histogram of mitochondria size $\left(\mathrm{nm}^{2}\right) n>100$, using at least five different cells of each condition CTRL $(n=2)$ and IPF $(n=2)$. (C) Images were semi-automatic analyzed using the ImageJ software. The length of mitochondria was used to determine mitochondrial elongation mean per image; at least 70 mitochondria per condition were measured.
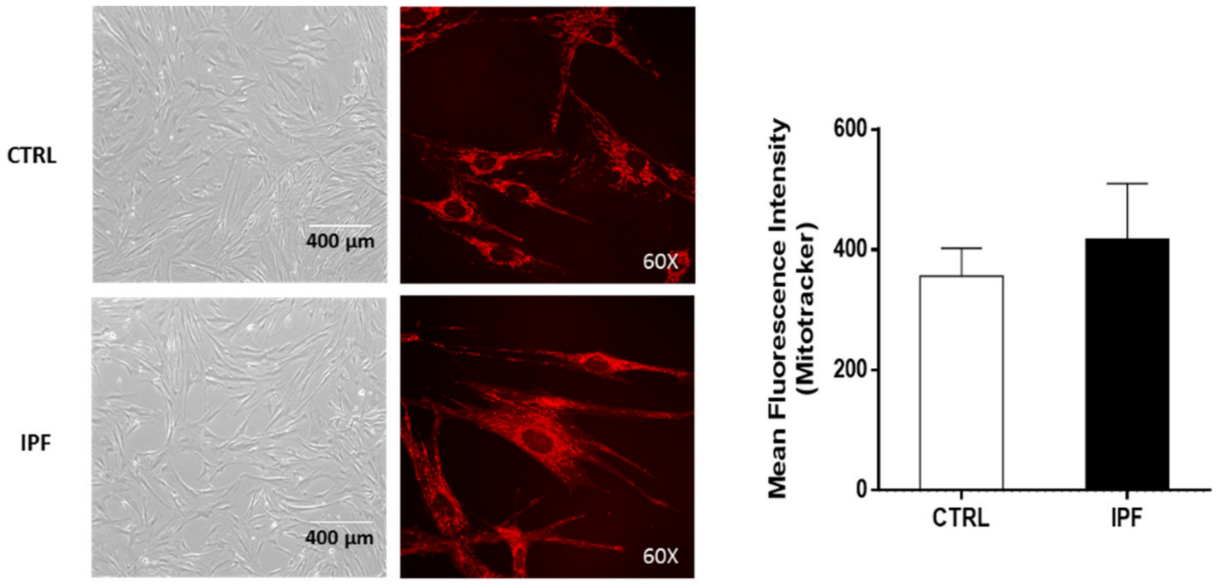

Figure 10. Idiopathic pulmonary fibrosis fibroblasts do not show differences in mean fluorescence intensity of MitoTracker compared to control fibroblasts (whole cells). To determine indirectly if IPF fibroblasts had a lower number of mitochondria than control fibroblasts, using a confocal microscope, we performed an analysis of the mean total fluorescence intensity of MitoTracker in a Z-stack of complete fibroblasts. The results are expressed as the mean \pm SEM. The differences were analyzed using an unpaired $t$-test, ${ }^{*} p<0.05$ vs. control. CTRL $(n=2)$ and $\operatorname{IPF}(n=2)$.

\section{Discussion}

A growing body of evidence demonstrates that IPF fibroblasts are resistant to apoptosis [7-10]. The mechanisms are unclear, but evidence indicates the participation of the extrinsic pathway of apoptosis, such as the inhibition of Fas-mediated apoptotic cell 
death [8]. However, no studies have investigated the role of the critical intrinsic pathway of apoptosis mediated by mitochondria. In this context, our study demonstrates that IPF fibroblasts show resistance to mPTP opening, low cytochrome c levels, mitochondrial dysfunction, and a fragmented mitochondrial network. Recently, mitochondrial dysfunction has been reported in IPF fibroblasts associated with the induction and maintenance of the senescent phenotype [27], but its specific role in the apoptosis of these cells is uncertain. Mitochondria-induced apoptosis arises from various internal cell stresses, resulting in the release of cytochrome $\mathrm{c}$ toward the cytosol. The above is a crucial process in the intrinsic apoptosis pathway and is related to the $\mathrm{MPTP}$ opening. Once in the cytosol, cytochrome c activates the apoptosome formation, which is a cytosolic multiprotein complex composed of cytochrome c, Apaf-1, and ATP. This complex induces the caspase 9 activation, triggering the effector caspases activation that provokes cell death [28]. Therefore, in the fibrotic process, the dysregulation of cytochrome $c$ release can result in the accumulation of apoptosis-resistant fibroblasts, excessive extracellular matrix deposition, and disruption of the lung architecture.

In this work, we demonstrated that IPF fibroblasts presented a marked decrease in basal cytochrome c levels and its release after stimulation with mitomycin C. cytochrome $c$ is a crucial signaling molecule during apoptosis, but it also plays an essential role in oxidative phosphorylation by transferring electrons from complex III to complex IV of the electron transport chain [29]. A substantial portion of cytochrome $\mathrm{c}$ is cardiolipinassociated, embedded in mitochondrial cristae, while the remaining cytochrome $\mathrm{c}$ is free in the intramembrane space. The release of cytochrome $\mathrm{c}$ from mitochondria is carried out during the early apoptosis stages and requires a two-step process. First, the solubilization of cytochrome $\mathrm{c}$ involves a breaking of the electrostatic and hydrophobic forces that it usually maintains with cardiolipin. Second, the mitochondrial permeability transition is sufficient to release cytochrome $c$ into the cytosol [30]. Thus, the decrease in basal cytochrome $c$ levels observed in fibroblasts from patients with IPF may be related to decreased electron transfer, oxygen consumption, and ATP synthesis. In this context, various studies have reported that the lack of cytochrome c disrupts the assembly and stability of respiratory complexes I and IV in fibroblasts [31].

Interestingly, embryonic fibroblasts derived from mice deficient in the somatic isoform of cytochrome $\mathrm{c}$ are resistant to apoptosis by agents known to trigger the intrinsic apoptotic pathway. This effect was associated with respiratory chain defects [32]. The mitochondrial apoptosis pathway is regulated mainly by changes in the permeability of the mitochondrial membrane or by alterations in its function. Mitochondrial outer membrane permeabilization is the ultimate step of apoptotic signal transduction pathways, which converge on mitochondria. $\mathrm{mPTP}$ is one of the representative systems proposed to be responsible for mitochondrial outer membrane permeabilization [33]. Although the concept of the MPTP is still evolving, mounting evidence indicates that the MPTP is directly responsible for cytochrome c release. For this reason, it is considered a strategic regulator of cell death. The mPTP is a non-specific conductance channel in the inner mitochondrial membrane that allows the flux of metabolites with a molecular weight of up to $1.5 \mathrm{kDa}$.

Induction of the MPTP leads to mitochondrial depolarization, inhibition of ATP synthesis, $\mathrm{Ca}^{2+}$ uptake, respiratory inhibition, generation of ROS, mitochondrial swelling, and potentially the rupture of the outer mitochondrial membrane leading to release of mitochondrial apoptogenic proteins such as cytochrome c, Smac/DIABLO, endonuclease G, and AIF. Therefore, it is not surprising that studies of the MPTP attract substantial attention. However, despite significant effort, the exact molecular composition of the mPTP is still a matter of debate.

The mPTP opening can be induced by high concentrations of mitochondrial $\mathrm{Ca}^{2+}$, oxidative stress, and Pi and can be inhibited by cyclosporin $\mathrm{A}$, adenine nucleotides, $\mathrm{Mg}^{2+}$, acidic $\mathrm{pH}$, and reducing agents in the cells [16]. The MPTP has been implicated in several diseases, but to our knowledge, no studies had been performed on IPF fibroblasts. We demonstrated for the first time that IPF fibroblasts are resistant to ionomycin-induced mPTP 
opening; this is an intriguing finding, because high basal ROS production by IPF fibroblasts has been reported [34], and an MPTP opening should be expected as a consequence of high mitochondrial ROS production. However, a similar effect has been reported in cancer cells. For example, Norman et al. demonstrated that mPTP inhibition by cyclosporin A promotes skin cancer in transplanted patients by allowing keratinocyte survival under conditions of genotoxic stress, highlighting the critical role of MPTP inhibition in tumor development [35].

On the other hand, different factors can influence this resistance to ionomicyn-induced mPTP opening, such as the expression of protein components. Recently, it has been proposed that in addition to the ANT, VDAC, cyclophilin D, the ATP synthase is part of the $\mathrm{MPTP}[17,18]$.

However, this idea has been questioned in recent studies, where some authors showed that $\mathrm{mPTP}$ persists in the absence of several subunits of ATP synthase $[19,20]$. We analyzed the expression levels of ATP synthase by WB and did not find significant differences between control and IPF fibroblasts, suggesting that the content of ATP synthase cannot influence the function of mPTP. Interestingly, when we analyzed the expression of ANT, we found that this protein shows decreased levels in IPF fibroblasts, which could suggest its participation in the mPTP inhibition observed in IPF fibroblasts.

Diverse studies show that drugs that stabilize the conformation of ANT in the cytosol enhanced mPTP opening, whereas others that stabilize ANT in the matrix inhibited MPTP opening by decreasing the sensitivity to $\left[\mathrm{Ca}^{2+}\right]$. When the ANT is stabilized in the cytosolic conformation by carboxyattrayloside, it provides a structural basis for mPTP formation by increasing sensibility to $\left[\mathrm{Ca}^{2+}\right]$. While on the other hand, bongkrekic acid, an inhibitor of ANT that stabilizes the matrix conformation, inhibits mPTP opening by decreasing the sensitivity to $\left[\mathrm{Ca}^{2+}\right][36]$. This effect was observed in our results when using attrayloside or bongkrekic acid in the control lung fibroblasts. We observed an increase in apoptosis when stimulated fibroblasts with attrayloside and inhibition with bongkrekic acid.

Other factors that influence the opening of the MPTP may be related to cytochrome c interaction with lipids of the inner mitochondrial membrane or the capacity to accumulate calcium in the mitochondria, as calcium is a well-established mPTP activator. The reduction of mitochondrial calcium release induces MPTP inhibition.

Inhibition of the mPTP augments $\mathrm{Ca}^{2+}$ accumulation in mitochondria, stabilizes mitochondrial membrane potential, and defers $\mathrm{Ca}^{2+}$ dysregulation [36].

Interestingly, our results on mitochondrial calcium quantification show a marked decrease in mitochondria calcium release in patients with IPF, confirming that mPTP activity decreases in these cells. In addition, we did not observe mitochondrial potential membrane changes, which support that $\mathrm{mPTP}$ is inhibited in IPF fibroblasts.

Opening of the MPTP might also stimulate autophagy to eliminate abnormal mitochondria. Thus, the mitochondrial outer membrane recruits the autophagy proteins ATG5 and LC3, not only for mitophagy but also for the anchorage and share of the lipid moieties required for the elongation of the initial phagophore [37]. Interestingly, we have previously found that IPF fibroblasts show a decrease in autophagy caused by activation of the mechanistic target of rapamycin complex 1 (mTORC1) pathway, contributing to the resistance to cell death [38].

As mentioned, we also showed that stimulus with BKA inhibited the mitomycininduced cell death in control fibroblasts, while the MPTP inductor ATR renders the cell more susceptible to cell death. BKA prevents acidification and is a ligand for the ANT that can inhibit apoptosis. Our results concur with Lui et al., who demonstrated that inhibition of mPTP by cyclosporine A (CsA) and BKA affected p53 translocation in mitochondria, leading to protection against the loss of mitochondrial membrane potential and complex I activity and eventually suppression of apoptosis [39].

We also found that IPF fibroblasts show mitochondrial dysfunction, as evidenced by decreased oxygen consumption and decreased ATP production, although we did not observe significant changes in the ratio ADP/ATP and mitochondrial membrane potential. 
The fact that the ADP / ATP ratio does not change suggests that the cell adapts to maintain a minimal mitochondrial activity to supply enough ATP and keep the cell alive with basal transmembrane potential, avoiding cell death through the intrinsic pathway of apoptosis.

These findings agree with those previously reported by Álvarez et al. [40], who found low ATP levels in senescent IPF fibroblasts, which were associated with decreased OCR, diminished glycolytic capacity, and abnormalities in oxidative phosphorylation $[28,41]$.

Considerable evidence indicates that the mitochondrial network organization is associated directly with the bioenergetic function and ROS generation. In this sense, our results showed that IPF fibroblasts exhibited a fragmented mitochondrial network and scarce, thinned, and disordered mitochondria with a few electrodense cristae. Diverse studies have shown that cristae architecture is determined by cristae-shaping proteins and depends on the dimeric state of the ATP-synthase [42]. The formation of mitochondrial cristae increases the mitochondrial surface, locating a higher number of enzyme complexes in the mitochondria, improving oxidative phosphorylation (OXPHOS). Alterations in respiration and changes in ATP levels might be associated with mitochondrial morphology modulators. Benard et al. [43] demonstrated that the silencing of DRP1 in HeLa cells resulted in alterations of the mitochondrial network morphology. This effect was associated with reducing sensitivity to apoptosis inducers, potent inhibition of energy production, abnormal connectivity, and multiple budding areas, which suggested a perturbation of mitochondrial dynamics.

Furthermore, we observed a reduction in coupled respiration and ATP production. Studies with the effect of OXPHOS inhibition on mitochondrial network organization in primary human fibroblasts suggest that it may contribute to these abnormalities. Thus, the formation of vesicles due to the inhibition of complex I by rotenone can be regarded as a direct consequence of impaired OXPHOS function [43]. In general, a highly efficient OXPHOS correlates with a highly interconnected and ramified network and enlarged cristae compartments, whereas low OXPHOS activity and high glycolysis correlate with bulkier, more spherical tubules, and isolated mitochondria, displaying reduced intra-cristae space.

We found alterations in the respiratory chain, but we cannot describe it exactly. Therefore, we propose that it is necessary to study the formation of mitochondrial complexes and respirasomes because there is evidence supporting the fact that cytochrome $\mathrm{c}$ can influence the function of the chain. Furthermore, morphology observed in IPF fibroblasts increases the evidence that there are issues with the respiratory chain, since the mitochondrial ridges exhibit lesser electro-density than in control fibroblasts. The relationship between mitochondrial morphology and function has gained significant attention in recent years, and it has been verified that there is a direct relationship between the cristae structure and the respiratory chain.

Mitochondria are very complex organelles that control many diverse functions that, as a whole, define the fate of the cell, and mitochondrial dysfunction is usually understood as an event leading to cell death. However, we found that, on the contrary, mitochondrial dysfunction is associated with resistance to apoptosis in IPF fibroblasts, as it occurs in cancer cells. Therefore, the study of the in-depth mechanisms through which mitochondria dysfunction leads to proliferation or death in different pathologies will be helpful to identify new therapeutic targets against IPF, cancer, or other pathologies.

Tissue fibrosis is likely an evolutionarily conserved adaptive process, and persistent fibrosis almost always accompanies incomplete or unsuccessful regeneration. However, in this case, the underlying mechanisms that contribute to the persistence of myofibroblasts and continuous extracellular matrix accumulation in fibrotic tissues remain poorly understood. A growing body of evidence indicates that one of them is the evasion of apoptosis, which usually occurs during physiological wound healing. However, the molecular pathways that are involved in the apoptosis resistance of myofibroblast in fibrotic tissues are still unclear and may even involve different mechanisms. Some evidence indicates the participation of the extrinsic pathway of apoptosis, but no studies had investigated the role of the intrinsic pathway mediated by mitochondria. 
In this context, we thought that changes in the mPTP opening, given its relevance in regulating cell death, could be involved. Our results revealed that MPTP is inhibited in IPF fibroblasts, and that calcium, a well-established activator of $\mathrm{mPTP}$, is decreased and pro-apoptotic proteins such as cytochrome $\mathrm{c}$ are released as well. We consider that this finding is relevant to understand some of the biopathological mechanisms that participate in the apoptosis resistance of fibroblasts during progressive fibrosis as in IPF. A better understanding of the molecular composition of the MPTP and apoptosis mechanisms will provide clues for effective and selective therapeutic strategies for the treatment of this devastating disease.

\section{Materials and Methods}

\subsection{Reagents}

We purchased the Cell Proliferation Reagent WST-1 (11644807001) from Roche Diagnostics, Mannheim, Germany. The FITC Annexin V Apoptosis Detection Kit (556547) was from BD Biosciences, San Diego, CA, USA. The Mitochondria Isolation Kit for Cultured Cells (89874), Pierce ${ }^{\mathrm{TM}}$ BCA Protein Assay Kit (23225), HRP-anti-goat IgG (611620), and Anti-GAPDH (PA1-987) were from Thermo Fisher Scientific, Rockford, IL, USA. The Image-IT $^{\mathrm{TM}}$ LIVE Mitochondrial Transition Pore Assay Kit (I35103), MitoProbe ${ }^{\mathrm{TM}}$ Transition Pore Assay Kit (M34153), ATP Determination Kit (A22066), and tetraethyl benzimidazolyl carbocyanine iodide dye JC-1 (T3168) were from Molecular Probes, Eugene, OR, USA. The ADP / ATP ratio assay kit (MAK135), anti- $\beta$-actin (A5441), oligomycin A, rotenone, antimycin A, L-glutamic acid, L( )-malic acid disodium salt, sodium succinate dibasic hexahydrate, carbonyl cyanide 4-(trifluoromethoxy) phenylhydrazine (FCCP), carbonyl cyanide-3chlorophenyl hydrazone (CCCP), ethylene glycol-bis (2-aminoethylether)- $\mathrm{N}, \mathrm{N}^{\prime} \mathrm{N}^{\prime}, \mathrm{N}^{\prime}$-tetra acetic acid (EGTA), potassium salt, 4-(2-hydroxyethyl)piperazine-1-ethanesulfonic acid potassium salt (HEPES), bovine serum albumin (BSA), free fatty acid, magnesium chloride $(\mathrm{MgCl})$, potassium phosphate monobasic $(\mathrm{KH} 2 \mathrm{PO} 4)$, cytochrome $\mathrm{c}$, bongkrekic acid (BKA), and atractyloside (ATR) were purchased from Sigma-Aldrich from St. Louis, MO, USA. Mitomycin C (sc-3514); anti-VDAC-1 (sc-390996), and anti-caspase-3 (sc-7272) were from Santa Cruz Biotechnology, Heidelberg, Germany. Anti-cytochrome c (612504) and HRP-anti-rabbit IgG (406401) were from BioLegend, San Diego, CA, USA. The anti-caspase9 (ab32539) was from Abcam Cambridge, MA, USA. HRP-Anti-Mouse IgG (115-035-003) was acquired from Jackson Immuno Research, West Grove, PA, USA.

\subsection{Bioethics and Experimental Design}

The Bioethics Committee of the Instituto Nacional de Enfermedades Respiratorias approved this research, with ethic approval code B05-21, (20 September 2018). Fibroblasts from IPF patients were obtained from lung biopsies performed for diagnostic purposes; all participants provided informed consent, and their personal and identification data were protected. As controls, healthy lung fibroblasts were obtained by lobectomy from patients without morphological data of interstitial disease, and the commercial human normal fibroblasts line NHLF (Normal Human Lung Fibroblasts CC-2512 Lonza Clonetics) was used. Healthy and IPF lung fibroblasts matched by age were studied under basal conditions and after mitomycin C-induced apoptosis.

\subsection{Cell Culture}

The fibroblasts were cultured in Ham medium (F-12), supplemented with penicillin $(100 \mathrm{U} / \mathrm{mL})$, streptomycin $(100 \mathrm{mg} / \mathrm{mL})$, and 10\% fetal bovine serum (SFB), and incubated in a humid atmosphere at $37{ }^{\circ} \mathrm{C}$ with $5 \% \mathrm{CO}_{2}$, until $80 \%$ confluence. Fibroblasts were treated with different doses of mitomycin $C(10,25$, or $50 \mu \mathrm{g} / \mathrm{mL})$ in F-12 medium with $1 \%$ SFB at different times $(4,8,16$, and $24 \mathrm{~h})$. Control fibroblasts were incubated in F-12 medium with 1\% SFB for the same period. After the treatment, fibroblasts were washed three times with phosphate-buffered saline (PBS) and kept in the F-12 medium for subsequent experiments. 


\subsection{Cell Viability}

Cell viability was assessed using the Cell Proliferation Reagent WST-1 assay following the manufacturer's instructions. Cells were seeded in 96-well plates at a final density of $5 \times 10^{3}$ cells per well in $100 \mu \mathrm{L}$ of culture medium. After treatment with mitomycin-C, $10 \mu \mathrm{L}$ of WST-1 solution was added to each well, and the cells were incubated for $3 \mathrm{~h}$ at $37^{\circ} \mathrm{C}$ in a $5 \% \mathrm{CO}_{2}$ atmosphere. The optical density was measured using a multimodal microplate reader (Synergy HTX BioTek, Winooski, VT, USA) at a wavelength of $450 \mathrm{~nm}$.

\subsection{Cell Apoptosis}

Fibroblasts were stimulated with mitomycin-C for $24 \mathrm{~h}$ and stained with Annexin V-IP for flow cytometric analysis. Staurosporine $(1 \mu \mathrm{M})$ was used as a positive control. Cells were acquired using a FACSAria flow cytometer (BD Biosciences). Data were analyzed using FlowJo 7.8 software (FlowJo, Ashland, OR, USA, Becton Dickinson and Company).

\subsection{Preparation of the Cytosolic and Mitochondrial Fractions}

Control and IPF fibroblasts were cultured in a cell culture flask of $175 \mathrm{~cm}^{2}$ until $80 \%$ confluence (approximately two or three flasks by experimental condition). Fibroblasts were stimulated with mitomycin C ( $25 \mu \mathrm{g} / \mathrm{mL})$ for 4 and $24 \mathrm{~h}$. After treatment with mitomycin $\mathrm{C}$, the cells were washed and trypsinized, and their viability was analyzed by a Trypan Blue exclusion test. For each experimental condition, $1 \times 10^{7}$ viable cells were used.

The isolation of mitochondrial and cytosolic fractions of fibroblast cultures was performed with the Mitochondria Isolation Kit for Cultured Cells, following the manufacturer's instructions. For this assay, we used a Dounce homogenizer to disrupt cells, and subsequently, we separated the fractions by differential centrifugation. The mitochondrial and cytosolic fractions were used for analysis by Western blotting. The mitochondrial pellet was lysed with $50 \mu \mathrm{L}$ of $2 \%$ detergent 3-(3-cholamidopropyl) dimethylammonio)-1propane sulfonate (CHAPS) in Tris buffer saline (TBS; $25 \mathrm{mM}$ Tris, $0.15 \mathrm{M} \mathrm{NaCl} ; \mathrm{pH}$ : 7.2). The supernatant contained the soluble mitochondrial protein that was quantified by the Pierce BCA Protein Assay Kit.

\subsection{Western Blotting}

Cytosolic and mitochondrial fractions and cell lysates were analyzed by Western blot. Cellular lysates from human normal and IPF fibroblasts were prepared using radioimmunoprecipitation assay buffer (RIPA) with phenylmethylsulfonyl fluoride (PMSF) and protease inhibitors. The protein concentration was quantified using the Bradford assay. Briefly, $16 \mu \mathrm{g}$ of protein were separated by Sodium Dodecyl Sulfate-Polyacrylamide SDS-PAGE ( $110 \mathrm{~V}$ for $1 \mathrm{~h}$ at room temperature) and transferred onto a nitrocellulose membrane using a wet chamber blotting system. The nitrocellulose membranes were blocked with $5 \%$ skim milk for $1 \mathrm{~h}$ and afterwards incubated overnight at $4{ }^{\circ} \mathrm{C}$ with the primary antibodies: anti-cytochrome c (1:500); anti-caspase 9 (1:500); anti-VDAC-1 (1:100); anti-Bax (1:100); anti-ATP5A (1:500); anti-ANT1 (1:1000); anti-GAPDH (1:2000); anti- $\beta$-actin $(1: 10,000)$. The secondary antibodies were incubated for $1 \mathrm{~h}$ at room temperature with the following dilutions: anti-mouse (1:10,000), anti-rabbit (1:2500), and anti-goat (1:1000).

\section{8. cytochrome c Release}

The cytosolic fraction was filtered through a polyvinylidene fluoride membrane PVDF $(0.45 \mu \mathrm{M})$, and the cytochrome c content was determined by high-performance liquid chromatography (HPLC) using a $300 \AA$ Aelta Pack C4 column with $5 \mu \mathrm{M}$ particles $(3.9 \times 150 \mathrm{~mm}$; Waters). A gradient of $30 \%$ acetonitrile in water with trifluoroacetic acid $(0.1 \% \mathrm{vol} / \mathrm{vol})$ to $70 \%$ acetonitrile in water with trifluoroacetic acid $(0.1 \% \mathrm{vol} / \mathrm{vol})$ over $15 \mathrm{~min}$ with a flow rate of $1 \mathrm{~mL} / \mathrm{min}$ was used as was described elsewhere [44]. cytochrome c was detected at $393 \mathrm{~nm}$, and its concentration was quantified using a standard curve of cytochrome $\mathrm{c}$ from bovine heart. 


\section{9. mPTP Opening}

The mPTP opening was assessed by cobalt quenching of calcein-AM fluorescence. After treatment, cells were loaded with calcein-AM $(1 \mu \mathrm{M}$, Molecular Probes, Life Technologies) at $37^{\circ} \mathrm{C}$ in the dark, $\mathrm{CoCl}_{2}(1 \mathrm{mM})$ was added, and cells were incubated for another $15 \mathrm{~min}$. Then, the fluorescence of 30,000 cells for each experiment was measured with a flow cytometer (FACS Aria II, BD, San Jose, CA, USA), and the data were processed with the FlowJo software. For imaging experiments, $1 \times 10^{4}$ fibroblasts were cultured on glassbottom culture dishes. First, cells were loaded with calcein-AM $(1 \mu \mathrm{M})$ and MitoTracker Red $(150 \mathrm{nM})$, with or without $\mathrm{CoCl}_{2}(1 \mathrm{mM})$, in Hanks' Balanced Salt Solution (HBSS) $1 \mathrm{X}$ for $15 \mathrm{~min}$ at $37^{\circ} \mathrm{C}$. Then, cells were washed three times with HBSS 1X. Live images of the cells were captured with the Olympus FluoView FV1000 Confocal Microscope and analyzed using the ImageJ software version 1.53i (Bethesda, MD, USA).

\subsection{Intracellular Calcium Concentration Assay $\left(\left[\mathrm{Ca}^{2+}\right]_{i}\right)$}

To evaluate $\left[\mathrm{Ca}^{2+}\right]_{\mathrm{i}}$ changes induced by trifluoromethoxy carbonyl cyanide phenylhydrazone (FCCP) in control and IPF fibroblasts, cells were plated in round coverslips coated with poly-L-Lysine and cultured for 2 days. Then, cells were loaded with Fura 2-AM $(2.5 \mu \mathrm{M})$ in a low concentration of $\mathrm{Ca}^{2+}(0.1 \mathrm{mM})$ and at room temperature. Then, fibroblasts were incubated for $1 \mathrm{~h}$ at $37{ }^{\circ} \mathrm{C}$ under a $5 \% \mathrm{CO}_{2}$ atmosphere. Afterward, cells were transferred to a heated perfusion chamber $\left(37^{\circ} \mathrm{C}\right)$ mounted on an inverted Nikon Diaphot 200 microscope (Nikon, Tokyo, Japan). Cells were recorded under continuous perfusion and carbogen bubbling (to maintain $\mathrm{pH}$ at 7-4) at a rate of $2-2.5 \mathrm{~mL} / \mathrm{min}$ with Krebs-Ringer buffer ( $\mathrm{NaCl} 118 \mathrm{mM}, \mathrm{NaHCO}_{3} 25 \mathrm{mM}, \mathrm{KCl} 4.7 \mathrm{mM}, \mathrm{KH}_{2} \mathrm{PO}_{4} 1.2 \mathrm{mM}$, $\mathrm{MgSO}_{3} 1.2 \mathrm{mM}$, glucose $11 \mathrm{mM}$, and $\mathrm{CaCl}_{2} 2.5 \mathrm{mM}$ ) at $37^{\circ} \mathrm{C}$. After the recording of basal fluorescence, cells were exposed to FCCP $1 \mu \mathrm{M}$. Next, fibroblasts loaded with Fura 2-AM were alternately submitted to Xe lamp at $340 \mathrm{~nm}$ and $380 \mathrm{~nm}$ excitation light, and the emission fluorescence was measured at $510 \mathrm{~nm}$ using a microphotometer (model D-104), from Photon Technology International (PTI, Princeton, NJ, USA). Fluorescence was measured at intervals of $0.5 \mathrm{~s}$ for $10 \mathrm{~min}$, and the intracellular $\mathrm{Ca}^{2+}$ concentration $\left(\left[\mathrm{Ca}^{2+}\right]_{\mathrm{i}}\right)$ was calculated according to the Grynkiewicz [45] formula as follows:

$$
\left[\mathrm{Ca}^{2+}\right]_{\mathrm{i}}=\mathrm{bKd}\left(\mathrm{R}-\mathrm{R}_{\min }\right) /\left(\mathrm{R}_{\max }-\mathrm{R}\right)
$$

where $\mathrm{Kd}$ is the dissociation constant of Fura-2AM, $\mathrm{b}$ is the ratio of fluorescent signals $(\mathrm{R})$ at $380 \mathrm{~nm}$ for $\mathrm{Ca}^{2+}$-free and $\mathrm{Ca}^{2+}$-saturated dye, $\mathrm{R}_{\min }$ is $\mathrm{R}$ in the absence of external $\mathrm{Ca}^{2+}$, and $R_{\max }$ is $\mathrm{R}$ in saturating $\left[\mathrm{Ca}^{2+}\right]_{i}[46]$. These parameters were determined in vitro. The mean 340-380 nm fluorescence ratios for $R_{\max }(6.06)$ and $R_{\min }(0.39)$ were obtained by exposing the cells to $\mathrm{Ca}^{2+}(10 \mathrm{mM})$ in the presence of ionomycin $(10 \mu \mathrm{M})$, and $\mathrm{Ca}^{2+}$ free Krebs with EGTA $(10 \mathrm{mM})$, respectively. The fluorescence ratio at $380 \mathrm{~nm}$ light excitation in $\mathrm{Ca}^{2+}$ free medium and $\mathrm{Ca}^{2+}$ saturated cells was 4.23. The Kd of Fura 2-AM was assumed to be $386 \mathrm{nM}$ [46]. To evaluate the $\mathrm{Ca}^{2+}$ response to FCCP, cells were perfused with $1 \mu \mathrm{M}$ FCCP to stimulate the mitochondrial permeability transition pore (mPTP). To pharmacologically determine if mPTP were involved in this response, a blocker cyclosporin A (CspA) $10 \mu \mathrm{M}$ plus ATP was used.

\subsection{Cell Respirometry}

\subsubsection{Respirometry in Non-Permeabilized Cells}

The oxygen consumption experiments in intact cells were performed as previously described using a high-resolution respirometry $\mathrm{O} 2 \mathrm{k}$ meter (Oroboros Instruments, Innsbruck, Austria) [47]. Briefly, measures were made at $37^{\circ} \mathrm{C}$ using $2 \mathrm{~mL}$ of culture medium. The respiratory parameters were defined as (a) basal respiration, corresponding to the oxygen consumption in the presence only of cells, (b) the leak of the respiration, corresponding to cellular oxygen consumption in the presence of $5 \mu \mathrm{M}$ oligomycin, (c) respiratory control corresponding to the ratio basal/leak, (d) the maximum uncoupled respiration was 
achieved by titrations of $0.5 \mu \mathrm{L}$ of $1 \mu \mathrm{M}$ CCCP. All parameters were normalized by the number of cells and corrected by the non-mitochondrial residual respiration, which was obtained by adding $5 \mu \mathrm{M}$ rotenone plus $5 \mu \mathrm{M}$ antimycin $\mathrm{A}$.

\subsubsection{Respirometry in Permeabilized Cells with Digitonin}

The oxygen consumption in permeabilized fibroblasts was performed as was previously described by Kuznetsov et al. [48]. Control and IPF fibroblasts were seeded in standard conditions, and the trypan blue exclusion test was used to determine cell density. For each experimental condition, $5 \times 10^{5}$ fibroblasts were added, and the system was equilibrated again for $5 \mathrm{~min}$. The reaction was carried out in respiration medium (EGTA $0.5 \mathrm{mM} ; \mathrm{MgCl}_{2} 3 \mathrm{mM} ; \mathrm{KH}_{2} \mathrm{PO}_{4} 10 \mathrm{mM}$; HEPES $20 \mathrm{mM}$; BSA $1 \mathrm{~g}$; mannitol $110 \mathrm{mM}$ for liter) $\mathrm{pH} 7.1$, adjusted with $5 \mathrm{~N} \mathrm{KOH}$. Next, the fibroblasts were permeabilized with digitonin $(20 \mu \mathrm{g} / \mathrm{mL})$ and incubated for $5 \mathrm{~min}$ at $37^{\circ} \mathrm{C}$. After the addition of digitonin, the respiration rate should markedly decline for $3-5 \mathrm{~min}$. Then, glutamate/malate $(10 \mathrm{mM} / 5 \mathrm{mM})$ was used as a substrate for complex I activity, ADP was added to induce ATP production, and the respiration was inhibited by $0.5 \mu \mathrm{M}$ rotenone, which is a specific inhibitor of complex I. Afterward, succinate $(10 \mathrm{mM})$ was used as a substrate to induce complex II supported respiration, ADP was added to induce ATP production, and respiration was inhibited with oligomycin $(5 \mu \mathrm{M})$ (state 4). Finally, $2 \mathrm{mM}$ ADP was added for maximal (state 3) mitochondrial respiration. The respiratory control ratio (RCR) was calculated by dividing state $3 /$ state 4 respiratory values.

\subsection{Mitochondrial Membrane Potential $(\Delta \psi m)$}

Mitochondrial $\Delta \psi$ was measured using JC-1, which is a fluorescent dye that exhibits potential-dependent accumulation in mitochondria. Polarized mitochondria are marked by punctate orange-red fluorescent staining. On depolarization, the orange-red punctate staining is replaced by diffuse green monomer fluorescence. Thus, mitochondrial depolarization is indicated by a decrease in the red/green fluorescence intensity ratio. Briefly, IPF and control fibroblasts were stained with JC-1 in Ham F-12 culture medium for 30 min at $37^{\circ} \mathrm{C}$ in the dark. Then, cells were washed twice with PBS and stimulated with the uncoupling agent FCCP $(100 \mu \mathrm{M})$ or oligomycin $(5 \mu \mathrm{M})$. Fibroblasts were observed with a confocal microscope using the 20x objective, and both green $(520 \mathrm{~nm})$ and red $(572 \mathrm{~nm})$ fluorescence were measured to detect the emission shift. This shift was calculated as the $\mathrm{red} /$ green fluorescence intensity ratio. Images were analyzed using ImageJ software.

\subsection{ATP Production}

ATP production was determined quantitatively in IPF and control fibroblasts using the ATP Determination Kit, which uses a bioluminescent assay. For this assay, 10,000/well cells were plated on a white 96-well microplate with a clear bottom, and the assay was performed following the manufacturer's instructions. The bioluminescence was recorded using a multimodal microplate reader (Synergy HTX BioTek, Winooski, VT, USA) in the luminometer mode.

\subsection{ADP/ATP Ratio}

The ADP / ATP ratio in IPF and control fibroblasts was measured using the ADP/ATP ratio assay kit. Briefly, IPF and control fibroblasts were plated at a density of $10,000 \mathrm{cells} / \mathrm{cm}^{2}$ in a white 96-well microplate with a clear bottom. Following overnight incubation, the ADP/ATP ratio was determined following the manufacturer's guidelines. Next, the bioluminescence was measured using a multimodal microplate reader (Synergy HTX BioTek, Winooski, VT, USA).

\subsection{Transmission Electron Microscopy (TEM)}

TEM was done with the support of the Center for Advanced Microscopy (Northwestern University). In brief, lung fibroblasts were cultured on Thermanox coverslips placed in 
a 24-well plate. After $48 \mathrm{~h}$ of incubation, samples were fixed in $0.1 \mathrm{M}$ sodium cacodylate ( $\mathrm{pH} 7.2$ ) containing $2 \%$ paraformaldehyde and $2.5 \%$ glutaraldehyde and post-fixed with $2 \%$ osmium tetroxide and stained with $3 \%$ uranyl acetate. Samples were dehydrated in ascending ethanol grades, transitioned with a 1:1 mixture of ethanol and resin, and embedded in the resin mixture of the EMbed-812 kit. Using a Leica Ultracut UC6 ultramicrotome, ultra-thin sections $(70 \mathrm{~nm})$ were collected on 200 mesh copper grids and post-stained with $3 \%$ uranyl acetate and Reynolds lead citrate. Ultra-thin sections were used to obtain images from each sample with the Tecnai G2 Spirit transmission electron microscope (FEI Company, Hillsboro, OR, USA) at $120 \mathrm{kV}$. The length of mitochondria was used to determine mitochondrial elongation mean per image; at least 70 mitochondria per condition were measured. Images were analyzed using the ImageJ software.

\subsection{Statistical Analysis}

All the data were expressed as the mean \pm standard error mean (SEM). Two-way or one-way analysis of variance (ANOVA) followed by Bonferroni test assessed the significance of the differences. To analyze the significant differences between the control and IPF group, we used an unpaired $t$-test. $p$-values of less than 0.05 were considered to be statistically significant. Statistical analysis was performed using the GraphPad Prism 5.01 software (San Diego, CA, USA).

Author Contributions: Conceptualization, M.E.H., M.O.-I., A.P. and M.S.; methodology, E.R.L.-G., C.B., O.E.A.-T., Y.R., E.F.-S., C.M.-M.; software, A.S.-A., E.R.L.-G., M.O.-I. and Y.R.; validation, M.S., E.R.L.-G., C.B., J.P.-C. and M.E.H.; formal analysis, M.S., E.R.L.-G., A.P., M.O.-I., V.C.; investigation, E.R.L.-G., C.B., A.S.-A., J.P.-C.; resources, M.M., J.P.-C., A.P. and M.S.; data curation, A.S.-A., O.E.A.-T., E.F.-S. and C.M.-M.; writing-original draft preparation, A.P., M.S., E.R.L.-G., M.O.-I. and M.E.H.; writing—review and editing, A.P., M.S., E.R.L.-G.; visualization, V.C., M.M., J.P.-C.; supervision, A.P., M.S., M.E.H., J.P.-C.; project administration, A.P., M.S.; funding acquisition, A.P., M.S. and M.M. All authors have read and agreed to the published version of the manuscript.

Funding: This research did not receive any specific grant from funding agencies in the public, commercial, or not-for-profit sectors.

Institutional Review Board Statement: The study was conducted according to the guidelines of the Declaration of Helsinki and was approved by the Bioethics Committee of Instituto Nacional de Enfermedades Respiratorias “Ismael Cosío Villegas" (protocol code (B05-21), 20 September 2018).

Informed Consent Statement: Fibroblasts from IPF patients were obtained from lung biopsies performed for diagnostic purposes; healthy lung fibroblasts were obtained by lobectomy from patients without morphological data of interstitial disease; all participants provided informed consent, and their personal and identification data were protected.

Data Availability Statement: All the data are included in the manuscript.

Acknowledgments: This paper constitutes a partial fulfillment of Erika Rubi Luis-Garcia to obtain the PhD "Doctor en Ciencias del Posgrado en Ciencias Biológicas Experimentales", Universidad Nacional Autónoma de México (UNAM). We thank chemist Damaris Romero Rodriguez (Instituto Nacional de Enfermedades Respiratorias "Ismael Cosío Villegas") for their continuous support and technical assistance in flow cytometry.

Conflicts of Interest: The authors declare no conflict of interest.

\section{References}

1. Raghu, G.; Remy-Jardin, M.; Myers, J.L.; Richeldi, L.; Ryerson, C.J.; Lederer, D.J.; Behr, J.; Cottin, V.; Danoff, S.K.; Morell, F.; et al. Diagnosis of Idiopathic Pulmonary Fibrosis. An Official ATS/ERS/JRS/ALAT Clinical Practice Guideline. Am. J. Respir. Crit. Care Med. 2018, 198, e44-e68. [CrossRef] [PubMed]

2. King, T.E.; Pardo, A.; Selman, M. Idiopathic pulmonary fibrosis. Lancet 2011, 378, 1949-1961. [CrossRef]

3. Selman, M.; Pardo, A. Revealing the pathogenic and aging-related mechanisms of the enigmatic idiopathic pulmonary fibro-sis: An integral model. Am. J. Respir. Crit. Care Med. 2014, 189, 1161-1172. [CrossRef] [PubMed]

4. Pardo, A.; Selman, M. The Interplay of the Genetic Architecture, Aging, and Environmental Factors in the Pathogenesis of Idiopathic Pulmonary Fibrosis. Am. J. Respir. Cell Mol. Biol. 2021, 64, 163-172. [CrossRef] [PubMed] 
5. $\quad$ Bueno, M.; Lai, Y.-C.; Romero, Y.; Brands, J.; Croix, C.M.S.; Kamga, C.; Corey, C.; Herazo-Maya, J.D.; Sembrat, J.; Lee, J.; et al. PINK1 deficiency impairs mitochondrial homeostasis and promotes lung fibrosis. J. Clin. Invest. 2015, 125, 521-538. [CrossRef]

6. Darby, I.A.; Zakuan, N.; Billet, F.; Desmoulière, A. The myofibroblast, a key cell in normal and pathological tissue repair. Cell Mol. Life Sci. 2016, 73, 1145-1157. [CrossRef]

7. Jiang, C.; Liu, G.; Cai, L.; Deshane, J.; Antony, V.; Thannickal, V.J.; Liu, R.M. Divergent regulation of ATII cell and fibroblast apoptosis by PAI-1 in lung fibrosis. Am. J. Pathol. 2021, 191, 1227-1239. [CrossRef]

8. Im, J.; Kim, K.; Hergert, P.; Nho, R.S. Idiopathic pulmonary fibrosis fibroblasts become resistant to Fas ligand-dependent apoptosis via the alteration of decoy receptor 3. J. Pathol. 2016, 240, 25-37. [CrossRef]

9. Ramos, C.; Montaño, M.; García-Álvarez, J.; Ruiz, V.; Uhal, B.D.; Selman, M.; Pardo, A. Fibroblasts from idiopathic pulmo-nary fibrosis and normal lungs differ in growth rate, apoptosis, and tissue inhibitor of metalloproteinases expression. Am. J. Respir. Cell Mol. Biol. 2001, 24, 591-598. [CrossRef]

10. Sisson, T.H.; Maher, T.M.; Ajayi, I.O.; King, J.E.; Higgins, P.D.; Booth, A.J.; Sagana, R.L.; Huang, S.K.; White, E.S.; Moore, B.; et al. Increased survivin expression contributes to apoptosis-resistance in IPF fibroblasts. Adv. Biosci. Biotechnol. 2012, 3, 657-664. [CrossRef]

11. Malsin, E.S.; Kamp, D.W. The mitochondria in lung fibrosis: Friend or foe? Transl. Res. 2018, 202, 1-23. [CrossRef]

12. Galluzzi, L.; Zamzami, N.; de La Motte Rouge, T.; Lemaire, C.; Brenner, C.; Kroemer, G. Methods for the assessment of mito-chondrial membrane permeabilization in apoptosis. Apoptosis 2007, 12, 803-813. [CrossRef] [PubMed]

13. Bernardi, P. Mitochondrial Transport of Cations: Channels, Exchangers, and Permeability Transition. Physiol. Rev. 1999, 79, 1127-1155. [CrossRef] [PubMed]

14. Briston, T.; Selwood, D.; Szabadkai, G.; Duchen, M.R. Mitochondrial Permeability Transition: A Molecular Lesion with Multiple Drug Targets. Trends Pharmacol. Sci. 2019, 40, 50-70. [CrossRef]

15. Crompton, M.; Virji, S.; Ward, J. Cyclophilin-D binds strongly to complexes of the voltage-dependent anion channel and the adenine nucleotide translocase to form the permeability transition pore. JBIC J. Biol. Inorg. Chem. 1998, 258, 729-735. [CrossRef] [PubMed]

16. Woodfield, K.; Rück, A.; Brdiczka, D.; Halestrap, A.P. Direct demonstration of a specific interaction between cyclophilin-D and the adenine nucleotide translocase confirms their role in the mitochondrial permeability transition. Biochem. J. 1998, 336, 287-290. [CrossRef]

17. Urbani, A.; Giorgio, V.; Carrer, A.; Franchin, C.; Arrigoni, G.; Jiko, C.; Abe, K.; Maeda, S.; Shinzawa-Itoh, K.; Bogers, J.F.M.; et al Purified F-ATP synthase forms a $\mathrm{Ca}^{2+}$-dependent high-conductance chan-nel matching the mitochondrial permeability transition pore. Nat. Commun. 2019, 1, 4341. [CrossRef]

18. Pinke, G.; Zhou, L.; Sazanov, L.A. Cryo-EM structure of the entire mammalian F-type ATP synthase. Nat. Struct. Mol. Biol. 2020, 27, 1077-1085. [CrossRef]

19. Carroll, J.; He, J.; Ding, S.; Fearnley, I.M.; Walker, J.E. Persistence of the permeability transition pore in human mitochondria devoid of an assembled ATP synthase. Proc. Natl. Acad. Sci. USA 2019, 116, 12816-12821. [CrossRef]

20. He, J.; Carroll, J.; Ding, S.; Fearnley, I.M.; Walker, J.E. Permeability transition in human mitochondria persists in the absence of peripheral stalk subunits of ATP synthase. Proc. Natl. Acad. Sci. USA 2017, 114, 9086-9091. [CrossRef]

21. Bernardi, P.; Di Lisa, F. The mitochondrial permeability transition pore: Molecular nature and role as a target in cardiopro-tection. J. Mol. Cell. Cardiol. 2015, 78, 100-106. [CrossRef]

22. Rasola, A.; Bernardi, P. The mitochondrial permeability transition pore and its adaptive responses in tumor cells. Cell Calcium 2014, 56, 437-445. [CrossRef] [PubMed]

23. Narita, M.; Shimizu, S.; Ito, T.; Chittenden, T.; Lutz, R.J.; Matsuda, H.; Tsujimoto, Y. Bax interacts with the permeability transition pore to induce permeability transition and cytochrome c release in isolated mitochondria. Proc. Natl. Acad. Sci. USA 1998, 95, 14681-14686. [CrossRef] [PubMed]

24. Crompton, M. The mitochondrial permeability transition pore and its role in cell death. Biochem. J. 1999, 341, 233-249. [CrossRef] [PubMed]

25. Yan, C.; Kong, D.; Ge, D.; Zhang, Y.; Zhang, X.; Su, C.; Cao, X. Mitomycin C induces apoptosis in rheumatoid arthritis fibro-blast-like synoviocytes via a mitochondrial-mediated pathway. Cell Physiol. Biochem. 2015, 35, 1125-1136. [CrossRef]

26. Sun, Y.; Ge, Y.; Fu, Y.; Yan, L.; Cai, J.; Shi, K.; Cao, X.; Lu, C. Mitomicin C induces fibroblasts apoptosis and reduces epidural fibrosis by regulating miR-200b and its targeting of RhoE. Eur. J. Pharmacol. 2015, 765, 198-208. [CrossRef] [PubMed]

27. Schuliga, M.; Pechkovsky, D.; Read, J.; Waters, D.W.; Blokland, K.; Reid, A.; Hogaboam, C.; Khalil, N.; Burgess, J.; Prêle, C.M.; et al. Mitochondrial dysfunction contributes to the senescent phenotype of IPF lung fibroblasts. J. Cell. Mol. Med. 2018, 22, 5847-5861. [CrossRef]

28. Cavalcante, G.C.; Schaan, A.P.; Cabral, G.F.; Santana-da-Silva, M.N.; Pinto, P.; Vidal, A.F.; Ribeiro-Dos-Santos, Â. A Cell's Fate: An Overview of the Molecular Biology and Genetics of Apoptosis. Int. J. Mol. Sci. 2019, 20, 4133. [CrossRef]

29. Schug, Z.; Gottlieb, E. Cardiolipin acts as a mitochondrial signalling platform to launch apoptosis. Biochim. Biophys. Acta (BBA) Biomembr. 2009, 1788, 2022-2031. [CrossRef]

30. Ott, M.; Robertson, J.D.; Gogvadze, V.; Zhivotovsky, B.; Orrenius, S. cytochrome c release from mitochondria proceeds by a two-step process. Proc. Natl. Acad. Sci. USA 2002, 99, 1259-1263. [CrossRef] 
31. Vempati, U.D.; Han, X.; Moraes, C.T. Lack of cytochrome c in Mouse Fibroblasts Disrupts Assembly/Stability of Respiratory Complexes I and IV. J. Biol. Chem. 2009, 284, 4383-4391. [CrossRef] [PubMed]

32. Vempati, U.D.; Diaz, F.; Barrientos, A.; Narisawa, S.; Mian, A.M.; Millán, J.L.; Boise, L.; Moraes, C.T. Role of cytochrome c in Apoptosis: Increased Sensitivity to Tumor Necrosis Factor Alpha Is Associated with Respiratory Defects but Not with Lack of cytochrome c Release. Mol. Cell. Biol. 2007, 27, 1771-1783. [CrossRef]

33. Mathupala, S.P.; Ko, Y.H.; Pedersen, P.L. Hexokinase II: Cancer's double-edged sword acting as both facilitator and gate-keeper of malignancy when bound to mitochondria. Oncogene 2006, 25, 4777-4786. [CrossRef] [PubMed]

34. Bocchino, M.; Agnese, S.; Fagone, E.; Svegliati, S.; Grieco, D.; Vancheri, C.; Gabrielli, A.; Sanduzzi, A.; Avvedimento, E.V. Reactive Oxygen Species Are Required for Maintenance and Differentiation of Primary Lung Fibroblasts in Idiopathic Pulmonary Fibrosis. PLoS ONE 2010, 5, e14003. [CrossRef] [PubMed]

35. Norman, K.G.; Canter, J.A.; Shi, M.; Milne, G.L.; Morrow, J.D.; Sligh, J.E. Cyclosporine A suppresses keratinocyte cell death through MPTP inhibition in a model for skin cancer in organ transplant recipients. Mitochondrion 2010, 10, 94-101. [CrossRef]

36. Brustovetsky, $\mathrm{N}$. The role of adenine nucleotide translocase in the mitochondrial permeability transition. Cells 2020, 12, 2686. [CrossRef]

37. Ghavami, S.; Shojaei, S.; Yeganeh, B.; Ande, S.R.; Jangamreddy, J.R.; Mehrpour, M.; Christoffersson, J.; Chaabane, W.; Moghadam, A.R.; Kashani, H.H.; et al. Autophagy and apoptosis dysfunction in neurodegen-erative disorders. Prog. Neurobiol. 2014, 112, 24-49. [CrossRef]

38. Romero, Y.; Bueno, M.; Ramirez, R.; Álvarez, D.; Sembrat, J.C.; Goncharova, E.A.; Rojas, M.; Selman, M.; Mora, A.L.; Pardo, A. mTORC1 activation decreases autophagy in aging and idiopathic pulmonary fibrosis and contributes to apoptosis re-sistance in IPF fibroblasts. Aging Cell 2016, 15, 1103-1112. [CrossRef] [PubMed]

39. Liu, J.; Clair, D.K.S.; Gu, X.; Zhao, Y. Blocking mitochondrial permeability transition prevents p53 mitochondrial transloca-tion during skin tumor promotion. FEBS Lett. 2008, 582, 1319-1324. [CrossRef]

40. Álvarez, D.; Cárdenes, N.; Sellares, J.; Bueno, M.; Corey, C.; Hanumanthu, V.S.; Peng, Y.; D’Cunha, H.; Sembrat, J.; Nouraie, M.; et al. IPF lung fibroblasts have a senescent phenotype. Am. J. Physiol. Cell. Mol. Physiol. 2017, 313, L1164-L1173. [CrossRef] [PubMed]

41. Bueno, M.; Calyeca, J.; Rojas, M.; Mora, A.L. Mitochondria dysfunction and metabolic reprogramming as drivers of idiopathic pulmonary fibrosis. Redox Biol. 2020, 33, 101509. [CrossRef] [PubMed]

42. Paumard, P.; Vaillier, J.; Coulary, B.; Schaeffer, J.; Soubannier, V.; Mueller, D.M.; Brèthes, D.; di Rago, J.P.; Velours, J. The ATP synthase is involved in generating mitochondrial cristae morphology. EMBO J. 2002, 21, 221-230. [CrossRef] [PubMed]

43. Benard, G.; Bellance, N.; James, D.; Parrone, P.; Fernandez, H.; Letellier, T.; Rossignol, R. Mitochondrial bioenergetics and structural network organization. J. Cell Sci. 2007, 120, 838-848. [CrossRef] [PubMed]

44. Ruiz-Ramírez, A.; Barrios-Maya, M.A.; López-Acosta, O.; Molina-Ortiz, D.; El-Hafidi, M. cytochrome c release from rat liver mitochondria is compromised by increased saturated cardiolipin species induced by sucrose feeding. Am. J. Physiol. Endocrinol. Metab. 2015, 309, E777-E786. [CrossRef]

45. Grynkiewicz, G.; Poenie, M.; Tsien, R.Y. A new generation of Ca2+ indicators with greatly improved fluorescence properties. J. Biol. Chem. 1985, 260, 3440-3450. [CrossRef]

46. Kajita, J.; Yamaguchi, H. Calcium mobilization by muscarinic cholinergic stimulation in bovine single airway smooth muscle. Am. J. Physiol. Content Lung Cell. Mol. Physiol. 1993, 264, L496-L503. [CrossRef]

47. Doerrier, C.; Garcia-Souza, L.F.; Krumschnabel, G.; Wohlfarter, Y.; Mészáros, A.T.; Gnaiger, E. High-Resolution FluoRespi-rometry and OXPHOS Protocols for Human Cells, Permeabilized Fibers from Small Biopsies of Muscle, and Isolated Mito-chondria. Methods Mol. Biol. 2018, 1782, 31-70.

48. Kuznetsov, A.V.; Veksler, V.; Gellerich, F.N.; Saks, V.; Margreiter, R.; Kunz, W. Analysis of mitochondrial function in situ in permeabilized muscle fibers, tissues and cells. Nat. Protoc. 2008, 3, 965-976. [CrossRef] [PubMed] 